\title{
Complex Organic Molecules toward Embedded Low-mass Protostars
}

\section{Citation}

Bergner, Jennifer B., Karin I. Öberg, Robin T. Garrod, and Dawn M. Graninger. 2017. Complex Organic Molecules toward Embedded Low-mass Protostars. The Astrophysical Journal 841, no. 2: 120.

\section{Permanent link}

http://nrs.harvard.edu/urn-3:HUL.InstRepos:41668567

\section{Terms of Use}

This article was downloaded from Harvard University's DASH repository, and is made available under the terms and conditions applicable to Open Access Policy Articles, as set forth at http:// nrs.harvard.edu/urn-3:HUL.InstRepos:dash.current.terms-of-use\#OAP

\section{Share Your Story}

The Harvard community has made this article openly available.

Please share how this access benefits you. Submit a story.

Accessibility 
DRAFT VERSION MAY 16, 2017

Preprint typeset using $\mathrm{LAT}_{\mathrm{E}} \mathrm{X}$ style AASTeX6 v. 1.0

\title{
COMPLEX ORGANIC MOLECULES TOWARDS EMBEDDED LOW-MASS PROTOSTARS
}

\author{
Jennifer B. Bergner ${ }^{1}$, Karin I. Öberg ${ }^{2}$, Robin T. Garrod ${ }^{3}$, Dawn M. Graninger ${ }^{2}$
}

\footnotetext{
${ }^{1}$ Harvard University Department of Chemistry and Chemical Biology, Cambridge, MA 02138, USA

${ }^{2}$ Harvard-Smithsonian Center for Astrophysics, Cambridge, MA 02138, USA

${ }^{3}$ University of Virginia Departments of Chemistry and Astronomy, Charlottesville, VA 22904, USA
}

\section{ABSTRACT}

Complex organic molecules (COMs) have been observed towards several low-mass young stellar objects (LYSOs). Small and heterogeneous samples have so far precluded conclusions on typical COM abundances, as well as the origin(s) of abundance variations between sources. We present observations towards 16 deeply embedded (Class 0/I) low-mass protostars using the IRAM 30m telescope. We detect $\mathrm{CH}_{2} \mathrm{CO}, \mathrm{CH}_{3} \mathrm{CHO}, \mathrm{CH}_{3} \mathrm{OCH}_{3}, \mathrm{CH}_{3} \mathrm{OCHO}, \mathrm{CH}_{3} \mathrm{CN}, \mathrm{HNCO}$, and $\mathrm{HC}_{3} \mathrm{~N}$ towards $67 \%$, $37 \%, 13 \%, 13 \%, 44 \%, 81 \%$, and $75 \%$ of sources respectively. Median column densities derived using survival analysis range between $6.0 \times 10^{10} \mathrm{~cm}^{-2}\left(\mathrm{CH}_{3} \mathrm{CN}\right)$ and $2.4 \times 10^{12} \mathrm{~cm}^{-2}\left(\mathrm{CH}_{3} \mathrm{OCH}_{3}\right)$ and median abundances range between $0.48 \%\left(\mathrm{CH}_{3} \mathrm{CN}\right)$ and $16 \%$ ( $\left.\mathrm{HNCO}\right)$ with respect to $\mathrm{CH}_{3} \mathrm{OH}$. Column densities for each molecule vary by about one order of magnitude across the sample. Abundances with respect to $\mathrm{CH}_{3} \mathrm{OH}$ are more narrowly distributed, especially for oxygen-bearing species. We compare observed median abundances with a chemical model for low-mass protostars and find fair agreement, although some modeling work remains to bring abundances higher with respect to $\mathrm{CH}_{3} \mathrm{OH}$. Median abundances with respect to $\mathrm{CH}_{3} \mathrm{OH}$ in LYSOs are also found to be generally comparable to observed abundances in hot cores, hot corinos, and massive young stellar objects. Compared with comets, our sample is comparable for all molecules except $\mathrm{HC}_{3} \mathrm{~N}$ and $\mathrm{CH}_{2} \mathrm{CO}$, which likely become depleted at later evolutionary stages.

\section{INTRODUCTION}

Complex organic molecules (COMs), hydrogen-rich molecules with 6 or more atoms, have been observed towards high- and low-mass star forming regions, molecular outflows, and prestellar cores (e.g. Blake et al. 1987; Fayolle et al. 2015; Bottinelli et al. 2004b, 2007; Arce et al. 2008; Öberg et al. 2010; Bacmann et al. 2012; Cernicharo et al. 2012). COMs formed at these early stages of star formation can become incorporated into protoplanetary disks (Visser et al. 2009, 2011) and further into planetesimals and planets, seeding nascent planets with complex organic material. COM abundances around protostars are thus of considerable interest for the study of origins of life. Low-mass stars host most planetary systems, and so the molecular inventories towards low-mass young stellar objects (LYSOs) are most relevant for characterizing potentially habitable environments. Indeed, our own sun is a low-mass star, and observations of COMs towards LYSOs can inform our

Based on observations carried out under project numbers 00314 and 006-13 with the IRAM 30m Telescope. IRAM is supported by INSU/CNRS (France), MPG (Germany) and IGN (Spain) understanding of the protostellar phase of our own solar system, as well as the uniqueness of our solar system relative to others like it.

$\mathrm{COMs}$ are thought to mainly form within the ice mantles coating interstellar dust grains (Herbst \& van Dishoeck 2009). First, hydrogenation of atoms or small molecules forms small saturated $\mathrm{COMs}$ such as $\mathrm{CH}_{3} \mathrm{OH}$, so-called "zeroth-generation" species. Photolysis or radiolysis can then dissociate small molecules to form radicals (reviewed in Öberg 2016). During protostellar collapse the cloud material is heated, enabling diffusion and recombination of these small molecules and radicals to form larger complex molecules referred to as "firstgeneration" species. Modeling has shown that this becomes efficient above 30K (Garrod \& Herbst 2006). This mechanism produces the same types of molecules that are commonly observed in YSOs, although some COMs are still underproduced in models compared to observations (Caselli \& Ceccarelli 2012). Once temperatures reach 100-300K, large molecules desorb and react in the gas phase to form "second-generation" molecules. Gasphase reactions may also play a role in first-generation chemistry via the desorption of small molecules, namely $\mathrm{CH}_{3} \mathrm{OH}$, followed by reaction in the gas phase rather 
than on grain-surfaces (e.g. Balucani et al. 2015).

Both the ice and gas-phase chemistry scenarios predict a close connection between COMs and the original ice composition. Ice composition is known to vary between sources, especially $\mathrm{CH}_{3} \mathrm{OH}$ ice abundances (Öberg et al. 2011a). Furthermore, the chemistry may depend on the stellar radiation field and the evolutionary stage of the source in question. Observed variations in chemical richness may then signify inherent variations between different sources, or simply that objects are observed at different evolutionary stages, or a combination of both. Which source of variability dominates will affect predictions on the chemical environment in which planets form during later stages of star formation, and how much this can vary between different sources.

LYSOs are thought to undergo evolution from prestellar cores to protostellar envelopes, which are termed hot corinos once the center is warm enough to sublimate water ice (e.g. Caselli \& Ceccarelli 2012). COMs have been previously detected towards both envelopes and hot corinos of LYSOs (e.g. Cazaux et al. 2003; Bottinelli et al. 2004a, 2007; Öberg et al. 2011b). Öberg et al. (2014) combined these results from the literature with observations towards 6 young LYSOs and found that COM column densities and abundances span orders of magnitude between different sources. However, for several reasons it has been difficult to draw firm conclusions on typical abundances and the origin of variability between sources. These protostars span a range of evolutionary stages, from prestellar cores to evolved protostars with hot envelopes and no ice absorption. Moreover,the sample size is small ( 14 objects), and objects that were chosen for hosting interesting chemistry may not be representative of the true sample distribution.

Because of uncertainties in "typical" LYSO COM abundances, it is unclear how LYSOs compare with their massive counterparts (MYSOs) in terms of chemical richness. It has been claimed both that LYSOs are more enhanced in COMs than MYSOs (Bottinelli et al. 2007; Herbst \& van Dishoeck 2009) and also that LYSO abundances are comparable or smaller than MYSO abundances (Bisschop et al. 2008; Öberg et al. 2011b). A better estimate of typical COM abundances around LYSOs is required to resolve whether these protostars indeed host distinct chemistries.
To clarify both the characteristic chemistry of LYSOs, as well as to make meaningful comparisons with MYSOs, a homogeneous and unbiased sample is required. Here we present an extension of the pilot survey in Öberg et al. (2014), yielding a total of 16 low-mass protostars. Both the 6 previously observed sources and the 10 new sources are Class 0/I protostars, enabling a direct comparison of the chemistry between sources at a similar evolutionary stage.

In Section 2 we describe the observations and data reduction. In Section 3 we present the results, first deriving column densities and rotational temperatures for the observed COMs. We derive median values for COM abundances from this sample using survival analysis in order to estimate characteristic abundance frequencies, as well as correlations between different COM species. In Section 4, we describe a warm-up model used to simulate chemistry around LYSOs, and present the model results. In Section 5 we comment on implications for formation chemistry based on our findings. We also compare our observational results with the chemical model, and with previous studies of different classes of objects.

\section{OBSERVATIONS}

Source selection and observation strategy are described in detail in Graninger et al. (2016). Briefly, 16 Class 0/I YSOs, identified by their IR spectral indices, were selected from the Spitzer c2d ice sample presented in Boogert et al. (2008) based on their location in the northern hemisphere and their ice abundances (Table 1). The sources were observed with the IRAM 30m telescope using the EMIR $90 \mathrm{GHz}$ receiver and the Fourier Transform Spectrometer (FTS) backend. B1-a, B5 IRS1, L1489 IRS, IRAS 04108+2803, IRAS 03235+3004, and SVS 4-5 were observed June 1216, 2013 at $93-101 \mathrm{GHz}$ and $109-117 \mathrm{GHz}$. All other sources were observed on July 23-28, 2014 at 92 - 100 $\mathrm{GHz}$ and 108 - $116 \mathrm{GHz}$. All observations had a resolution of $200 \mathrm{kHz}$. The telescope beam size ranges from 27 " at $92 \mathrm{GHz}$ to 21 " at $117 \mathrm{GHz}$. Excluding the highest frequency spectral window, the rms values range from 2$7 \mathrm{mK}$; for each source, the rms around the $\mathrm{CH}_{3} \mathrm{CN} 60_{0}-5_{0}$ transition at $110.383 \mathrm{GHz}$ is listed in Table 1 to show the variability in rms between the sources observed.

Table 1. Source information of the complete 16-object c2d embedded protostar sample with ice detections

\begin{tabular}{|c|c|c|c|c|c|c|c|c|c|c|c|}
\hline Source & $\begin{array}{c}\text { R.A. } \\
\text { (J2000.0) }\end{array}$ & $\begin{array}{c}\text { Dec } \\
(\mathrm{J} 2000.0)\end{array}$ & Cloud & $\begin{array}{l}\mathrm{L}_{\mathrm{bol}} \\
\mathrm{L}_{\odot}\end{array}$ & $\begin{array}{l}\mathrm{M}_{\mathrm{env}} \\
\mathrm{M}_{\odot}\end{array}$ & $\alpha_{\mathrm{IR}}^{\mathrm{a}}$ & $\begin{array}{c}\mathrm{N}\left(\mathrm{CH}_{3} \mathrm{OH}\right) \\
10^{13} \mathrm{~cm}^{-2}\end{array}$ & $\begin{array}{c}\mathrm{N}\left(\mathrm{H}_{2} \mathrm{O}_{(\text {ice })}\right)^{\mathrm{a}} \\
10^{18} \mathrm{~cm}^{-2}\end{array}$ & $\begin{array}{c}\mathrm{X}_{\mathrm{CH}_{3} \mathrm{OH}(\mathrm{ice})}^{\mathrm{b}} \\
\% \mathrm{H}_{2} \mathrm{O}\end{array}$ & $\begin{array}{c}\mathrm{X}_{\mathrm{NH}_{3}(\text { ice })}^{\mathrm{b}} \\
\% \mathrm{H}_{2} \mathrm{O}\end{array}$ & $\begin{array}{c}\mathrm{rms} \\
(\mathrm{mK})\end{array}$ \\
\hline $\mathrm{B} 1-\mathrm{a}^{\mathrm{c}}$ & 03:33:16.67 & $31: 07: 55.1$ & Perseus & $1.3^{\mathrm{d}}$ & $2.8^{\mathrm{d}}$ & 1.87 & $10.21[3.24]$ & $10.39[2.26]$ & $<1.9$ & 3.33 [0.98] & 3.6 \\
\hline B1-c & 03:33:17.89 & 31:09:31.0 & Perseus & $3.7^{\mathrm{d}}$ & $17.7^{\mathrm{d}}$ & 2.66 & $1.69[0.51]$ & $29.55[5.65]$ & $<7.1$ & $<4.04$ & 5.5 \\
\hline
\end{tabular}


Table 1 (continued)

\begin{tabular}{|c|c|c|c|c|c|c|c|c|c|c|c|}
\hline Source & $\begin{array}{c}\text { R.A. } \\
\text { (J2000.0) }\end{array}$ & $\begin{array}{c}\text { Dec } \\
(\mathrm{J} 2000.0)\end{array}$ & Cloud & $\begin{array}{c}\mathrm{L}_{\text {bol }} \\
\mathrm{L}_{\odot}\end{array}$ & $\begin{array}{l}\mathrm{M}_{\mathrm{env}} \\
\mathrm{M}_{\odot}\end{array}$ & $\alpha_{\mathrm{IR}}^{\mathrm{a}}$ & $\begin{array}{c}\mathrm{N}\left(\mathrm{CH}_{3} \mathrm{OH}\right) \\
10^{13} \mathrm{~cm}^{-2}\end{array}$ & $\begin{array}{c}\mathrm{N}\left(\mathrm{H}_{2} \mathrm{O}_{(\text {ice })}\right)^{\mathrm{a}} \\
10^{18} \mathrm{~cm}^{-2}\end{array}$ & $\begin{array}{c}\mathrm{X}_{\mathrm{CH}_{3} \mathrm{OH}(\text { ice })}^{\mathrm{b}} \\
\% \mathrm{H}_{2} \mathrm{O}\end{array}$ & $\begin{array}{c}\mathrm{X}_{\mathrm{NH}_{3} \text { (ice) }}^{\mathrm{b}} \\
\% \mathrm{H}_{2} \mathrm{O}\end{array}$ & $\begin{array}{c}\mathrm{rms} \\
(\mathrm{mK})\end{array}$ \\
\hline B5 IRS $1^{\mathrm{c}}$ & $03: 47: 41.61$ & $32: 51: 43.8$ & Perseus & $4.7^{\mathrm{d}}$ & $4.2^{\mathrm{d}}$ & 0.78 & $1.77[0.46]$ & $2.26[0.28]$ & $<3.7$ & $<2.09$ & 7.0 \\
\hline HН 300 & 04:26:56.30 & $24: 43: 35.3$ & Taurus & $1.27^{\mathrm{e}}$ & $0.03^{f}$ & 0.79 & $0.24[0.10]$ & $2.59[0.25]$ & $<6.7$ & $3.46[0.90]$ & 5.8 \\
\hline IRAS $03235+3004^{c}$ & $03: 26: 37.45$ & $30: 15: 27.9$ & Perseus & $1.9^{\mathrm{d}}$ & $2.4^{\mathrm{d}}$ & 1.44 & $1.17[0.08]$ & $14.48[2.26]$ & $4.2[1.2]$ & $4.71[1.00]$ & 4.2 \\
\hline IRAS $03245+3002$ & 03:27:39.03 & $30: 12: 59.3$ & Perseus & $7.0^{\mathrm{d}}$ & $5.3^{\mathrm{d}}$ & 2.70 & $1.54[0.29]$ & $39.31[5.65]$ & $<9.8$ & $<4.40$ & 3.7 \\
\hline IRAS $03254+3050$ & $03: 28: 34.51$ & $31: 00: 51.2$ & Perseus & - & $0.3^{\mathrm{d}}$ & 0.90 & - & $3.66[0.47]$ & $<4.6$ & $6.66[1.37]$ & 3.9 \\
\hline IRAS $03271+3013$ & 03:30:15.16 & $30: 23: 48.8$ & Perseus & $0.8^{\mathrm{d}}$ & $1.2^{\mathrm{d}}$ & 2.06 & $0.42[0.04]$ & $7.69[1.76]$ & $<5.6$ & $6.37[1.86]$ & 4.8 \\
\hline IRAS $04108+2803^{c}$ & 04:13:54.72 & $28: 11: 32.9$ & Taurus & $0.62^{\mathrm{e}}$ & - & 0.90 & $1.04[0.44]$ & $2.87[0.4]$ & $<3.5$ & $4.29[1.03]$ & 4.0 \\
\hline IRAS $23238+7401$ & $23: 25: 46.65$ & $74: 17: 37.2$ & CB244 & - & - & 0.95 & $2.19[1.01]$ & $12.95[2.26]$ & $<3.6$ & $<1.24$ & 2.7 \\
\hline L1014 IRS & 21:24:07.51 & 49:59:09.0 & L1014 & - & - & 1.28 & $0.88[0.56]$ & $7.16[0.91]$ & $3.1[0.8]$ & $5.20[1.43]$ & 2.8 \\
\hline L1448 IRS1 & 03:25:09.44 & $30: 46: 21.7$ & Perseus & $17.0^{\mathrm{d}}$ & $16.3^{\mathrm{d}}$ & 0.34 & $0.23[0.04]$ & $0.47[0.16]$ & $<14.9$ & $<4.15$ & 3.7 \\
\hline L1455 IRS3 & 03:28:00.41 & 30:08:01.2 & Perseus & $0.32^{\mathrm{d}}$ & $0.2^{\mathrm{g}}$ & 0.98 & $1.46[0.88]$ & $0.92[0.37]$ & $<12.5$ & $6.21[3.51]$ & 3.9 \\
\hline L1455 SMM1 & $03: 27: 43.25$ & $30: 12: 28.8$ & Perseus & $3.1^{\mathrm{d}}$ & $5.3^{\mathrm{d}}$ & 2.41 & $1.48[0.76]$ & $18.21[2.82]$ & $<13.5$ & $<8.29$ & 4.1 \\
\hline L1489 IRS ${ }^{\mathrm{c}}$ & 04:04:43.07 & $26: 18: 56.4$ & Taurus & $3.7^{\mathrm{e}}$ & $0.1^{\mathrm{h}}$ & 1.10 & $0.69[0.14]$ & $4.26[0.51]$ & $4.9[1.5]$ & $5.42[0.96]$ & 5.4 \\
\hline SVS $4-5^{c}$ & $18: 29: 57.59$ & 01:13:00.6 & Serpens & $38^{\mathrm{i}}$ & - & 1.26 & 11.19 [4.29] & $5.65[1.13]$ & $25.2[3.5]$ & $\sim 4.3$ & 3.9 \\
\hline
\end{tabular}

Note-Adapted from Graninger et al. (2016)

a Boogert et al. (2008), ${ }^{b}$ Bottinelli et al. (2010), ${ }^{\text {c}}$ Sources were observed by Öberg et al. (2014), d Hatchell et al. (2007), e Furlan et al. (2008), ${ }^{\text {f Arce }}$

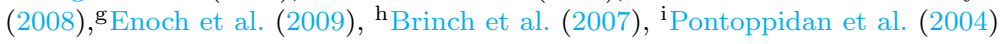

Spectra were reduced using CLASS $^{1}$. Global baselines were fit to each $4 \mathrm{GHz}$ spectral window using several line-free windows. Each individual scan was baseline subtracted and averaged. The beam efficiency was modified using Ruze's equation with scaling factor 0.861 and sigma of 63.6 microns, resulting in beam efficiencies at the first, middle, and last channel of 0.8106, 0.7975, and 0.7830. Together with a forward efficiency of 0.95 , the antenna temperature was converted to the main beam temperature $\mathrm{T}_{m b}$. Literature source velocities were used to convert spectra to rest frequency, with fine-tuning adjustments made with the $\mathrm{CH}_{3} \mathrm{OH} 2-1$ and $\mathrm{CN}$ 1-0 ladders.

\section{OBSERVATIONAL RESULTS}

\subsection{Molecule Detections}

Figure 1 shows the spectra of all observed sources. A wide dispersion in line richness is evident in the sample. B1-a and SVS 4-5 are very line-dense, followed by a collection of moderately rich sources: B1-c, IRAS 23238, L1455 IRS3, B5 IRS1 L1455 SMM1, IRAS 03235, L1014 IRS, IRAS 04108, and IRAS 03235. Finally, L1489, HH 300, IRAS 03271, IRAS 03253, and L1448 IRS1 are quite line-poor. A selection of complex organic molecules that have been observed towards other high- or low-mass pro-

\footnotetext{
${ }^{1}$ http://www.iram.fr/IRAMFR/GILDAS
}

tostars are covered by our spectral setting. We have focused on COMs that were detected towards several of the sources in our survey: $\mathrm{CH}_{3} \mathrm{CHO}, \mathrm{CH}_{3} \mathrm{OCH}_{3}$, $\mathrm{CH}_{3} \mathrm{OCHO}$, and $\mathrm{CH}_{3} \mathrm{CN}$. For comparison, we also include the smaller organics $\mathrm{HNCO}$ and $\mathrm{CH}_{2} \mathrm{CO}^{2}$, as well as the carbon chain cyanide $\mathrm{HC}_{3} \mathrm{~N}$. Line candidates within the observed frequency range were identified using the $\mathrm{JPL}^{3}$ and $\mathrm{CDMS}^{4}$ catalogs, limited by upper excitation energies less than $200 \mathrm{~K}$.

$\mathrm{CH}_{2} \mathrm{CO}, \mathrm{CH}_{3} \mathrm{CHO}, \mathrm{CH}_{3} \mathrm{OCH}_{3}, \mathrm{CH}_{3} \mathrm{OCHO}, \mathrm{CH}_{3} \mathrm{CN}$, $\mathrm{HNCO}$, and $\mathrm{HC}_{3} \mathrm{~N}$ are detected in $4,6,2,2,7,13$, and 12 sources respectively; this corresponds to detection percentages of $67 \%, 37 \%, 13 \%, 13 \%, 44 \%, 81 \%$, and $75 \%$ respectively. A molecule is considered to be detected provided that (1) at least one line with a $5 \sigma$ detection or two lines with $3 \sigma$ detections are observed, (2) there is no confusion with common interstellar or YSO molecular lines, and (3) non-detected lines have upper limits that are consistent with the populations predicted by detected lines. Upper limit treatments are discussed subsequently in more detail. Because even the line-rich sources in our survey are line-poor in comparison to hot

\footnotetext{
2 The $\mathrm{CH}_{2} \mathrm{CO}$ transition is only available for sources in the pilot survey: B1-a, B5 IRS1, L1489 IRS, IRAS 04108, IRAS 03235, and SVS 4-5

${ }^{3}$ http://spec.jpl.nasa.gov

${ }^{4}$ http://www.astro.uni-koeln.de/cdms/catalog
} 


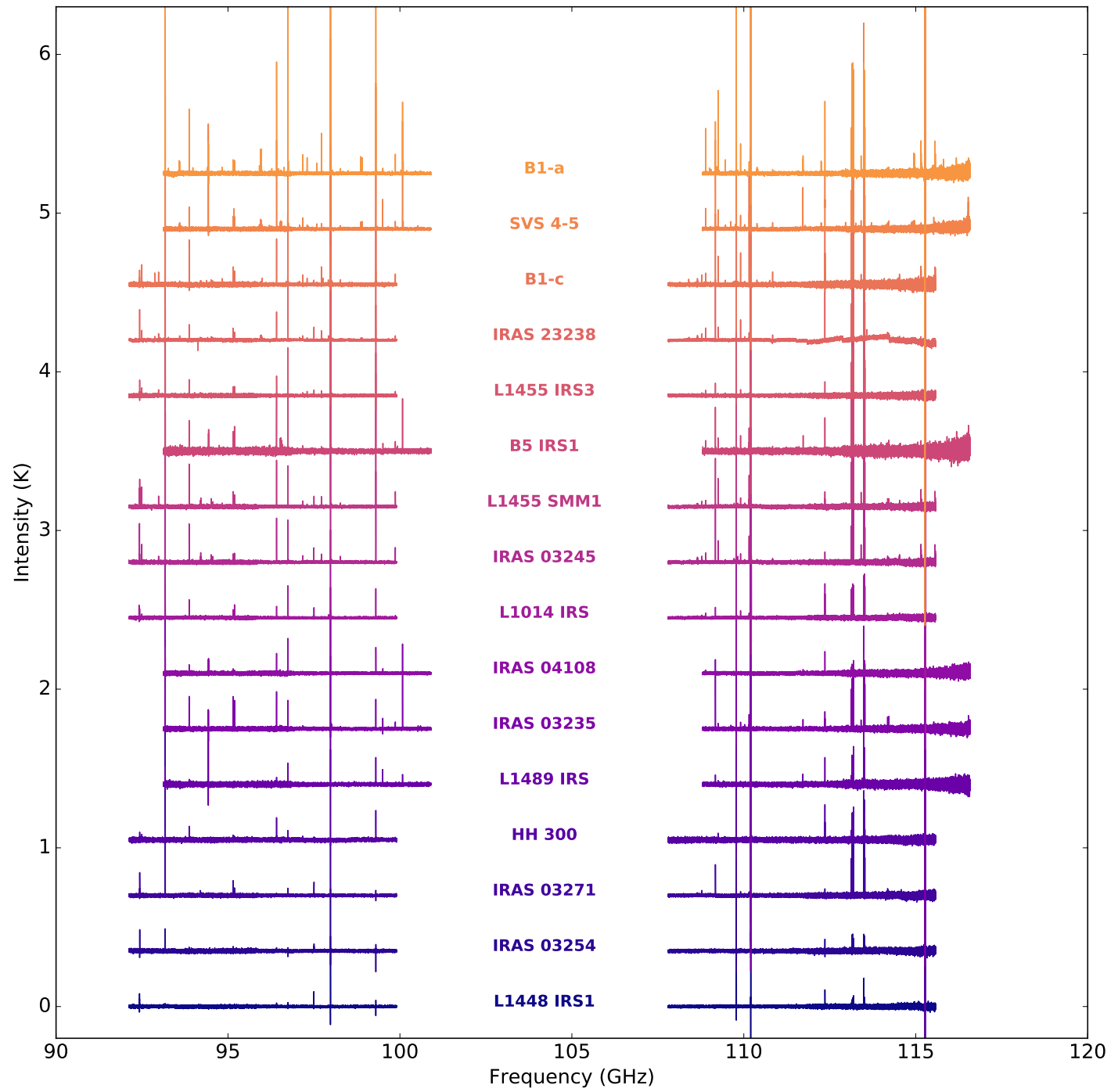

Figure 1. IRAM 30m spectra towards low-mass YSO sample, in order of line richness. 
cores, overlapping lines are generally not a concern, and a single line is sufficient to claim a detection when there are no competing line identifications.

Figure 2 shows the spectral window containing the $\mathrm{CH}_{3} \mathrm{CN}$ 6-5 ladder, with detections highlighted with a pink star. For all other molecules, spectral windows with detections are shown in the Appendix (Figures 15-20).

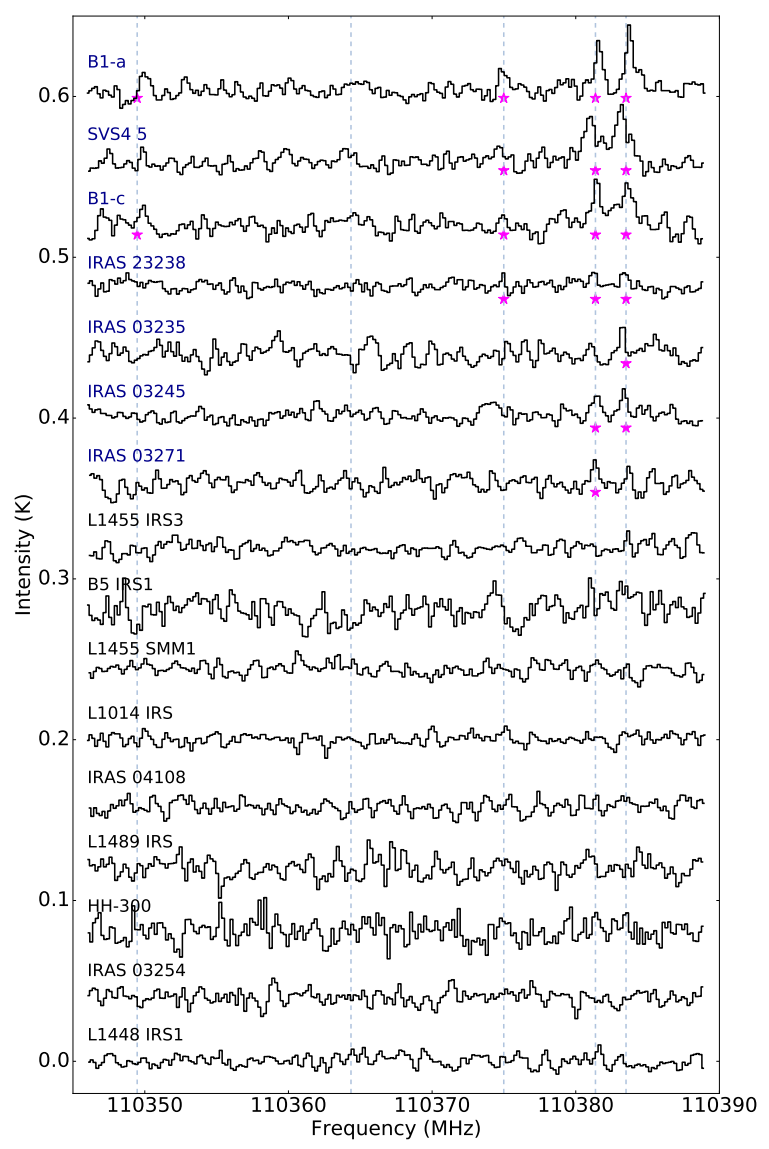

Figure 2. Blow-up of frequency range containing the $\mathrm{CH}_{3} \mathrm{CN}$ 6-5 ladder. Grey dashed lines show the line centers, sources with positive detections are written in blue font, and individual detected lines are highlighted with a pink star.

\subsection{Rotational Diagrams}

Integrated intensities for each observed line were determined by fitting single Gaussians to each feature, and are listed in Tables 6-12 in the Appendix. For lines with substantial wings, only the central peak was used in deriving column densities. Unresolved multiplets originating from the same species were treated as a single line by combining the degeneracies and line intensities. Overlapping lines with contributions from different species were excluded from further analysis. We assume a calibration uncertainty of $10 \%$.

For molecules with multiple line detections in a source we use rotational diagrams to calculate column densities (Goldsmith \& Langer 1999). The rotational diagram for
$\mathrm{CH}_{3} \mathrm{CHO}$ is shown in Figure 3, with all additional diagrams for detected molecules in the Appendix (Figures 21-26). Tables 2 and 3 summarize the resulting column densities and rotational temperatures. Rotational diagrams from the pilot survey were refit to include additional lines that were not identified in Öberg et al. (2014).

Upper level populations for each line are calculated by:

$$
\frac{N_{u}}{g_{u}}=\frac{3 k \int T_{m b} d V}{8 \pi^{3} \nu \mu^{2} S}
$$

Here, $N_{u}$ is the column density of molecules in the upper level, $g_{u}$ is the upper level degeneracy, $k$ is Boltzmann's constant, $\int T_{m b} d V$ is the integrated main beam temperature, $\nu$ is the transition frequency, $\mu$ is the dipole moment, and $S$ is the transition strength. Assuming optically thin lines and local thermodynamic equilibrium (LTE), each molecule's total column density $N_{T o t}$ and rotational temperature $T_{r o t}$ in each source can be determined from:

$$
\frac{N_{u}}{g_{u}}=\frac{N_{T o t}}{Q\left(T_{\text {rot }}\right)} e^{-E_{u} / T_{\text {rot }}}
$$

where $Q\left(T_{R o t}\right)$ is the partition function and $E_{u}$ is the energy of the upper level. We assume that all molecules within the beam can be described by a single temperature (e.g. Bisschop et al. 2007; Öberg et al. 2014; Fayolle et al. 2015).

For detected molecules, upper limits are calculated and included in the rotational diagrams for all listed transitions with an equal or greater transition strength and an upper level energy at most $15 \mathrm{~K}$ greater than the detected lines. $3 \sigma$ upper limits were calculated according to:

$$
\sigma=\mathrm{rms} \times \mathrm{FWHM} / \sqrt{n_{c h}}
$$

Here, the rms is taken from a $40 \mathrm{~km} \mathrm{~s}^{-1}$ spectral window containing the transition and FWHM is equal to the average FWHM of detected lines for that molecule in the same source. $n_{c h}$ is the number of channels across the FWHM, in this case equal to $\sim \mathrm{FWHM} / 0.6 \mathrm{~km} \mathrm{~s}^{-1}$ channel ${ }^{-1}$. Equation 1 is used to calculate the population upper limits.

In cases where a single transition was detected, the column densities were calculated adopting the average rotational temperatures in the sample for the species in question, if available. For $\mathrm{HNCO}$ and $\mathrm{CH}_{2} \mathrm{CO}$, at most one transition was detected for all sources. The average rotational temperature of $\mathrm{HC}_{3} \mathrm{~N}$ in our sample $(14 \mathrm{~K})$ was used for calculating $\mathrm{HNCO}$ column densities and that of $\mathrm{CH}_{3} \mathrm{CHO}(8 \mathrm{~K})$ used for calculating $\mathrm{CH}_{2} \mathrm{CO}$ column densities. This is based on spatial trends in emission observed in high-mass protostars (Bisschop et al. 2007; Öberg et al. 2013; Fayolle et al. 2015). 


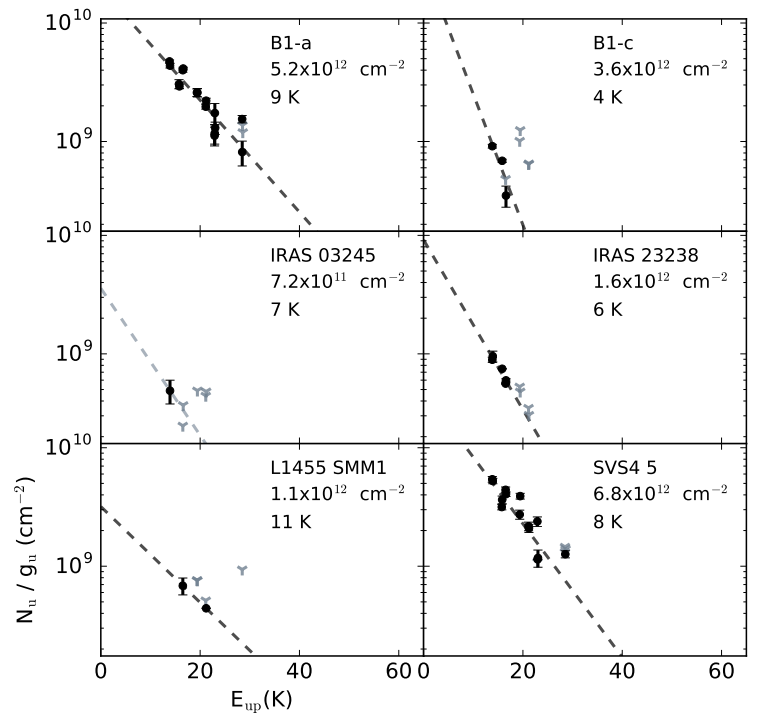

Figure 3. Rotation diagrams for $\mathrm{CH}_{3} \mathrm{CHO}$. Black circles indicate detections and grey triangles indicate upper limits. Black dashed lines represent the fits to the data. When a line could not be fit, a rotational temperature was assumed as described in the text, shown in grey dashed lines.
3. In this case, the rms for each non-detected molecule was taken from a $40 \mathrm{~km} \mathrm{~s}^{-1}$ spectral window containing its lowest-energy transition. We assume a FWHM equal to the average FWHM of all detected lines within a given source; for sources with no detections (HH-300 and L1448 IRS1) we assume the FWHM of $\mathrm{CH}_{3} \mathrm{OH}$ within the source. Upper limits for total column densities were calculated from equations 1 and 2 assuming the sampleaveraged rotational temperatures.

We note that B1-a and B1-c appear to have a detection of $\mathrm{CH}_{3} \mathrm{CN}_{65}-6_{4}$ at $110.349 \mathrm{GHz}$, which has an upper energy of $132.8 \mathrm{~K}$. We exclude these points from fitting the rotational diagram. The peak velocity of this transition is slightly offset from the other $\mathrm{CH}_{3} \mathrm{CN}$ detections for both sources in which we observe it; thus, if real this $\mathrm{CH}_{3} \mathrm{CN}$ line does not originate from the same location as the other lines. The line may trace $\mathrm{CH}_{3} \mathrm{CN}$ in a shock, but it is more likely due to an unidentified carrier; the latter was previously suggested by Bottinelli et al. (2007), who observed an excess of this $\mathrm{CH}_{3} \mathrm{CN}$ transition towards the hot corino NGC1333-IRAS4B.

For molecules with no detected transitions in a given source, a $3 \sigma$ upper limit was calculated using equation

Table 2. Column densities and rotational temperatures: Oxygen-bearing molecules

\begin{tabular}{|c|c|c|c|c|c|c|c|c|}
\hline & \multicolumn{2}{|c|}{$\mathrm{CH}_{2} \mathrm{CO}$} & \multicolumn{2}{|c|}{$\mathrm{CH}_{3} \mathrm{CHO}$} & \multicolumn{2}{|c|}{$\mathrm{CH}_{3} \mathrm{OCH}_{3}$} & \multicolumn{2}{|c|}{$\mathrm{CH}_{3} \mathrm{OCHO}$} \\
\hline & $\mathrm{N}_{T o t}\left(\mathrm{~cm}^{-2}\right)$ & $\mathrm{T}_{\text {Rot }}(\mathrm{K})$ & $\mathrm{N}_{T o t}\left(\mathrm{~cm}^{-2}\right)$ & $\mathrm{T}_{\text {Rot }}(\mathrm{K})$ & $\mathrm{N}_{T o t}\left(\mathrm{~cm}^{-2}\right)$ & $\mathrm{T}_{\text {Rot }}(\mathrm{K})$ & $\mathrm{N}_{\text {Tot }}\left(\mathrm{cm}^{-2}\right)$ & $\mathrm{T}_{\text {Rot }}(\mathrm{K})$ \\
\hline B1-a & $8.6(11.8) \times 10^{12}$ & 8 (2) & $5.2(0.8) \times 10^{12}$ & $9(1)$ & $8.1(1.3) \times 10^{12}$ & $22(5)$ & $5.9(6.2) \times 10^{12}$ & $17(11)$ \\
\hline B1-c & - & - & $3.6(3.4) \times 10^{12}$ & $4(1)$ & $<1.0 \times 10^{13}$ & $17(5)$ & $<9.5 \times 10^{12}$ & $16(1)$ \\
\hline B5 IRS1 & $<1.4 \times 10^{12}$ & $8(2)$ & $<1.1 \times 10^{12}$ & $8(2)$ & $<1.0 \times 10^{13}$ & $17(5)$ & $<9.4 \times 10^{12}$ & $16(1)$ \\
\hline НH-300 & - & - & $<4.5 \times 10^{11}$ & $8(2)$ & $<7.1 \times 10^{12}$ & $17(5)$ & $<6.5 \times 10^{12}$ & $16(1)$ \\
\hline IRAS 03235 & $1.0(1.4) \times 10^{12}$ & $8(2)$ & $<6.1 \times 10^{11}$ & $8(2)$ & $<6.4 \times 10^{12}$ & $17(5)$ & $<4.1 \times 10^{12}$ & $16(1)$ \\
\hline IRAS 03245 & - & - & $6.4(4.9) \times 10^{11}$ & $8(2)$ & $<5.6 \times 10^{12}$ & $17(5)$ & $<5.8 \times 10^{12}$ & $16(1)$ \\
\hline IRAS 03254 & - & - & $<6.4 \times 10^{11}$ & $8(2)$ & $<8.4 \times 10^{12}$ & $17(5)$ & $<5.3 \times 10^{12}$ & $16(1)$ \\
\hline IRAS 03271 & - & - & $<4.8 \times 10^{11}$ & $8(2)$ & $<8.5 \times 10^{12}$ & $17(5)$ & $<4.9 \times 10^{12}$ & $16(1)$ \\
\hline IRAS 04108 & $1.4(1.9) \times 10^{12}$ & 8 (2) & $<7.6 \times 10^{11}$ & 8 (2) & $<7.2 \times 10^{12}$ & $17(5)$ & $<6.0 \times 10^{12}$ & $16(1)$ \\
\hline IRAS 23238 & - & - & $1.6(1.0) \times 10^{12}$ & $6(2)$ & $<5.1 \times 10^{12}$ & $17(5)$ & $<3.7 \times 10^{12}$ & $16(1)$ \\
\hline L1014 IRS & - & - & $<3.1 \times 10^{11}$ & $8(2)$ & $<3.9 \times 10^{12}$ & $17(5)$ & $<3.2 \times 10^{12}$ & $16(1)$ \\
\hline L1448 IRS1 & - & - & $<4.2 \times 10^{11}$ & $8(2)$ & $<6.1 \times 10^{12}$ & $17(5)$ & $<4.1 \times 10^{12}$ & $16(1)$ \\
\hline L1455 IRS3 & - & - & $<4.3 \times 10^{11}$ & $8(2)$ & $<8.1 \times 10^{12}$ & $17(5)$ & $<4.8 \times 10^{12}$ & $16(1)$ \\
\hline L1455 SMM1 & - & - & $1.1(0.3) \times 10^{12}$ & $11(7)$ & $<6.7 \times 10^{12}$ & $17(5)$ & $<4.7 \times 10^{12}$ & $16(1)$ \\
\hline L1489 IRS & $<1.4 \times 10^{12}$ & 8 (2) & $<8.7 \times 10^{11}$ & $8(2)$ & $<8.1 \times 10^{12}$ & $17(5)$ & $<6.4 \times 10^{12}$ & $16(1)$ \\
\hline SVS4 5 & $9.0(12.4) \times 10^{12}$ & $8(2)$ & $6.8(1.1) \times 10^{12}$ & $8(1)$ & $1.2(0.5) \times 10^{13}$ & $12(3)$ & $7.0(4.4) \times 10^{12}$ & $15(5)$ \\
\hline
\end{tabular}

NotE-Uncertainties are listed in parentheses. $\mathrm{T}_{\text {Rot }}$ values are calculated from the rotational diagram method when possible; otherwise, sample-averaged values for that molecule are assumed, shown in italics in the table. 
Table 3. Column densities and rotational temperatures: Nitrogen-bearing molecules

\begin{tabular}{lllllll}
\hline \hline & \multicolumn{2}{c}{$\mathrm{CH}_{3} \mathrm{CN}$} & \multicolumn{2}{c}{$\mathrm{HC}_{3} \mathrm{~N}$} & \multicolumn{2}{c}{$\mathrm{HNCO}$} \\
& $\mathrm{N}_{\text {Tot }}\left(\mathrm{cm}^{-2}\right)$ & $\mathrm{T}_{\text {Rot }}(\mathrm{K})$ & $\mathrm{N}_{\text {Tot }}\left(\mathrm{cm}^{-2}\right)$ & $\mathrm{T}_{\text {Rot }}(\mathrm{K})$ & $\mathrm{N}_{\text {Tot }}\left(\mathrm{cm}^{-2}\right)$ & $\mathrm{T}_{\text {Rot }}(\mathrm{K})$ \\
\hline B1-a & $4.9(1.1) \times 10^{11}$ & $33(9)$ & $4.2(1.2) \times 10^{12}$ & $12(2)$ & $7.7(4.3) \times 10^{12}$ & $14(3)$ \\
B1-c & $3.5(0.6) \times 10^{11}$ & $18(2)$ & $4.2(3.4) \times 10^{12}$ & $14(3)$ & $8.8(4.9) \times 10^{12}$ & $14(3)$ \\
B5 IRS1 & $<1.7 \times 10^{11}$ & $27(7)$ & $3.2(1.2) \times 10^{12}$ & $11(2)$ & $3.5(2.0) \times 10^{12}$ & $14(3)$ \\
HH-300 & $<1.2 \times 10^{11}$ & $27(7)$ & $<1.3 \times 10^{11}$ & $14(3)$ & $<6.6 \times 10^{11}$ & $14(3)$ \\
IRAS 03235 & $1.6(0.9) \times 10^{11}$ & $27(7)$ & $3.9(1.0) \times 10^{12}$ & $13(2)$ & $1.8(1.0) \times 10^{12}$ & $14(3)$ \\
IRAS 03245 & $1.9(1.5) \times 10^{11}$ & $33(28)$ & $3.6(2.9) \times 10^{12}$ & $14(3)$ & $2.8(1.6) \times 10^{12}$ & $14(3)$ \\
IRAS 03254 & $<1.0 \times 10^{11}$ & $27(7)$ & $<9.2 \times 10^{10}$ & $14(3)$ & $<6.1 \times 10^{11}$ & $14(3)$ \\
IRAS 03271 & $1.9(1.1) \times 10^{11}$ & $27(7)$ & $1.7(1.4) \times 10^{12}$ & $14(3)$ & $1.4(0.8) \times 10^{12}$ & $14(3)$ \\
IRAS 04108 & $<1.2 \times 10^{11}$ & $27(7)$ & $<1.0 \times 10^{11}$ & $14(3)$ & $7.0(4.0) \times 10^{11}$ & $14(3)$ \\
IRAS 23238 & $1.4(0.3) \times 10^{11}$ & $33(6)$ & $3.2(2.6) \times 10^{12}$ & $14(3)$ & $4.6(2.6) \times 10^{12}$ & $14(3)$ \\
L1014 IRS & $<6.2 \times 10^{10}$ & $27(7)$ & $4.4(3.6) \times 10^{11}$ & $14(3)$ & $1.7(0.9) \times 10^{12}$ & $14(3)$ \\
L1448 IRS1 & $<9.4 \times 10^{10}$ & $27(7)$ & $<8.7 \times 10^{10}$ & $14(3)$ & $<4.4 \times 10^{11}$ & $14(3)$ \\
L1455 IRS3 & $<9.1 \times 10^{10}$ & $27(7)$ & $6.5(5.3) \times 10^{11}$ & $14(3)$ & $1.7(0.9) \times 10^{12}$ & $14(3)$ \\
L1455 SMM1 & $<1.1 \times 10^{11}$ & $27(7)$ & $2.4(1.9) \times 10^{12}$ & $14(3)$ & $2.4(1.3) \times 10^{12}$ & $14(3)$ \\
L1489 IRS & $<1.4 \times 10^{11}$ & $27(7)$ & $3.5(2.4) \times 10^{11}$ & $20(13)$ & $7.7(4.7) \times 10^{11}$ & $14(3)$ \\
SVS4 5 & $5.2(0.9) \times 10^{11}$ & $17(2)$ & $1.1(0.3) \times 10^{13}$ & $13(2)$ & $7.0(3.9) \times 10^{12}$ & $14(3)$ \\
\hline & & & & & &
\end{tabular}

Note-Uncertainties are listed in parentheses. $\mathrm{T}_{R o t}$ values are calculated from the rotational diagram method when possible; otherwise, sample-averaged values for that molecule are assumed, shown in italics in the table.

\subsection{Column densities and abundances}

3.3.1. COM detections and upper limits

Figure 4a shows the column densities derived for each molecule in all sources, both detections and upper limits. $\mathrm{CH}_{3} \mathrm{OCH}_{3}$ and $\mathrm{CH}_{3} \mathrm{OCHO}$ are each detected in only two sources. These detections are close to the upper limits for sources without detections. Thus, the upper limits for $\mathrm{CH}_{3} \mathrm{OCH}_{3}$ and $\mathrm{CH}_{3} \mathrm{OCHO}$ are not very constraining, and the variability in column density of these molecules cannot be well understood from this sample. All other molecules have multiple detections that are substantially larger than the non-detection upper limits, and the resulting ranges of derived column densities span at least an order of magnitude.

The sources B1-a and SVS 4-5, shown as the first and last bar in each grouping in Figure 4, stand out as being quite enhanced relative to the other sources. All 7 molecules are detected towards these sources, and the derived column densities are high compared to the sample medians. In contrast, the sources L1448 IRS1 and HH-300 have no detections. The majority of sources exhibit a range of moderate column densities, with a few but not all molecules detected in each source.

Abundances with respect to $\mathrm{CH}_{3} \mathrm{OH}$ are shown in
Figure 4b. $\mathrm{CH}_{3} \mathrm{OH}$ column densities are taken from Graninger et al. (2016). Because column densities of $\mathrm{CH}_{3} \mathrm{OH}$ are not available for IRAS 03254, we omit this source from comparison. Once normalized to $\mathrm{CH}_{3} \mathrm{OH}$, B1-a and SVS 4-5 molecule abundances are consistent or under-abundant compared with other sources, suggesting that their high column densities do not signify a distinct chemsitry.

\subsubsection{Median column densities and abundances}

Median abundances were first calculated using detections only; however, this neglects the information provided by upper limits, and thus risks over-estimating the median occurrences. Survival analysis using the KaplanMeier (KM) estimate of the survival function with left censorship was performed in order to account for this. In this method, all detections and non-detections are ordered, and then the values of positive detections are used to divide the total range of values into intervals. Upper limits within an interval are counted as having the lower delimiting value of the interval. In other words, each positive detection is weighted by the number of upper limits that occur between it and the next largest positive detection. Further details can be found in Feigelson \& Nelson (1985) and Miller et al. (1981). 


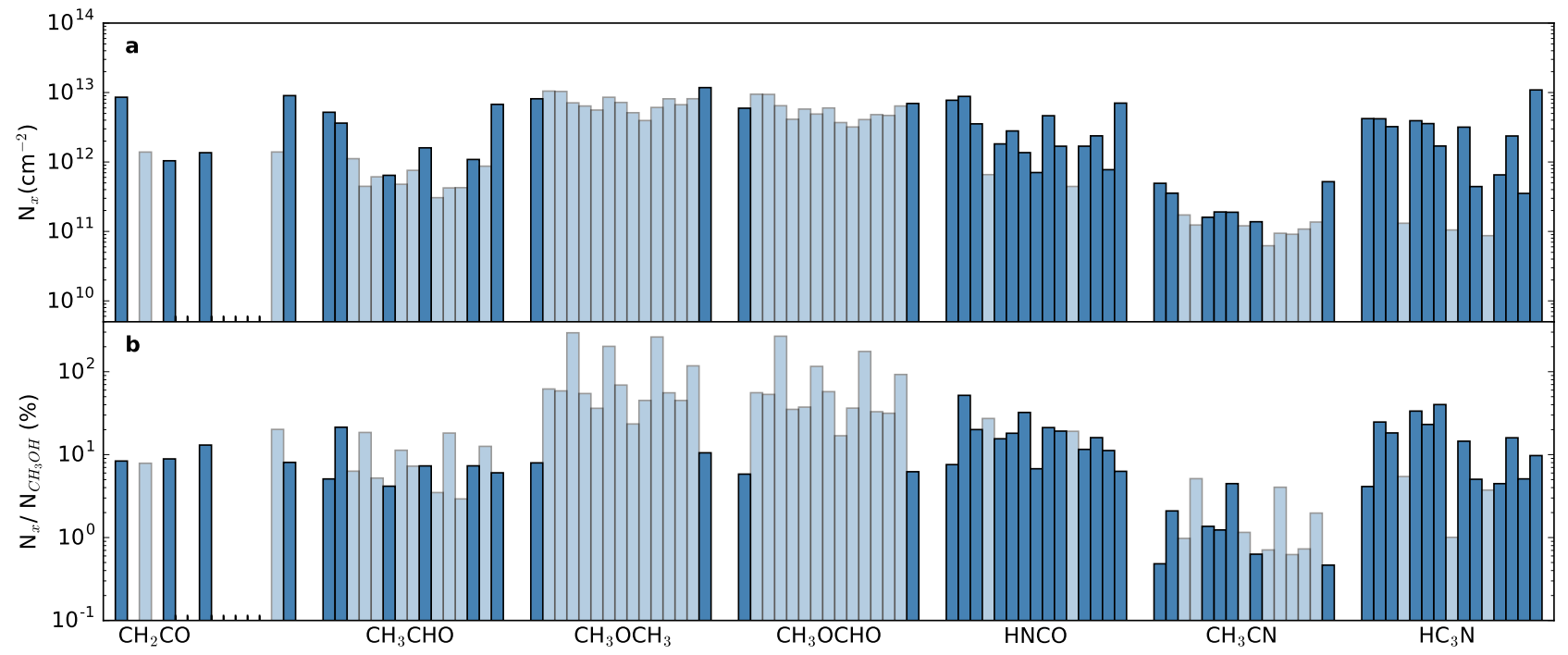

Figure 4. a: Observed column densities for each molecule. b: Abundances with respect to $\mathrm{CH}_{3} \mathrm{OH}$. For both panels, detections are shown in dark blue, and upper limits in light blue. For each molecule, sources are ordered alphabetically: B1-a, B1-c, B5 IRS1, HH-300, IRAS 03235, IRAS 03245, IRAS 03271, IRAS 04108, IRAS 23238, L1014 IRS, L1448 IRS1, L1455 IRS3, L1455 SMM1, L1489 IRS, SVS 4-5.

Table 4. Median column densities and abundances

\begin{tabular}{|c|c|c|c|}
\hline & \multicolumn{2}{|c|}{$\begin{array}{l}\text { Median column densities } \\
\qquad\left(10^{12} \mathrm{~cm}^{-2}\right)\end{array}$} & \multirow{2}{*}{$\begin{array}{c}\text { Median abundances } \\
\left(\% \text { with respect to } \mathrm{CH}_{3} \mathrm{OH}\right) \\
\text { Survival analysis }\end{array}$} \\
\hline & Detections only & Survival analysis ${ }^{\mathrm{a}}$ & \\
\hline $\mathrm{CH}_{2} \mathrm{CO}$ & 5.0 & $1.2 \stackrel{5.0}{0.78}$ & 8.28 .8 \\
\hline $\mathrm{CH}_{3} \mathrm{CHO}$ & 2.6 & $0.27 \stackrel{1.2}{0.14}$ & $4.4{ }_{2.3}^{6.1}$ \\
\hline $\mathrm{CH}_{3} \mathrm{OCH}_{3}$ & 9.9 & $2.4 \begin{array}{l}3.5 \\
1.2\end{array}$ & 5.39 .2 \\
\hline $\mathrm{CH}_{3} \mathrm{OCHO}$ & 6.5 & $1.9_{1.0}^{2.9}$ & $3.1 \underset{2.9}{6.0}$ \\
\hline $\mathrm{HNCO}$ & 2.4 & $1.8 \begin{array}{l}3.8 \\
0.76\end{array}$ & $16 \stackrel{20}{7.9}$ \\
\hline $\mathrm{CH}_{3} \mathrm{CN}$ & 0.19 & $0.060 \begin{array}{c}0.19 \\
0.03\end{array}$ & $0.48 \underset{0.46}{1.1}$ \\
\hline $\mathrm{HC}_{3} \mathrm{~N}$ & 3.2 & $2.0 \begin{array}{l}3.7 \\
0.29\end{array}$ & $7.4_{4.2}^{20}$ \\
\hline
\end{tabular}

${ }^{\text {a }}$ Lower and upper quartiles shown to the right of the median

Since the survivial function is discrete, median abundances are calculated by linear interpolation between the values above and below where the cumulative density function $(\mathrm{CDF})$ is equal to 0.5 . However, medians cannot be computed by the KM estimate for samples with only upper limits in the lowest $50 \%$ of values since the first positive detection occurs after the cumulative density has already exceeded 0.5. In this sample, this applies to $\mathrm{CH}_{3} \mathrm{OCH}_{3}, \mathrm{CH}_{3} \mathrm{OCHO}$, and $\mathrm{CH}_{3} \mathrm{CN}$. To mitigate this we calculate medians using the KM estimate with the lowest value assigned a "detection" status, regardless of its true identity as a detection or a non-detection. This may result in slightly elevated estimates for median values, but as seen in Figure 5 it is still a more realistic estimate of the median than using detections only.

Median column densities for each molecule are shown in Figure 5a and Table 4 for both methods. There is a clear difference between the medians calculated from only detections and those using survival analysis, demonstrating the importance of using the constraints provided by non-detections.

Median column densities of all species except $\mathrm{CH}_{3} \mathrm{CN}$ are on the order of $10^{12} \mathrm{~cm}^{-2}$, while $\mathrm{CH}_{3} \mathrm{CN}$ is over an order of magnitude lower. $\mathrm{CH}_{3} \mathrm{OCHO}$ and $\mathrm{CH}_{3} \mathrm{OCH}_{3}$ have among the highest median column densities compared to the other observed molecules, but this may be somewhat misleading since each is detected in only two sources. Given the large values of the partition function for these molecules, detections are possible only for 

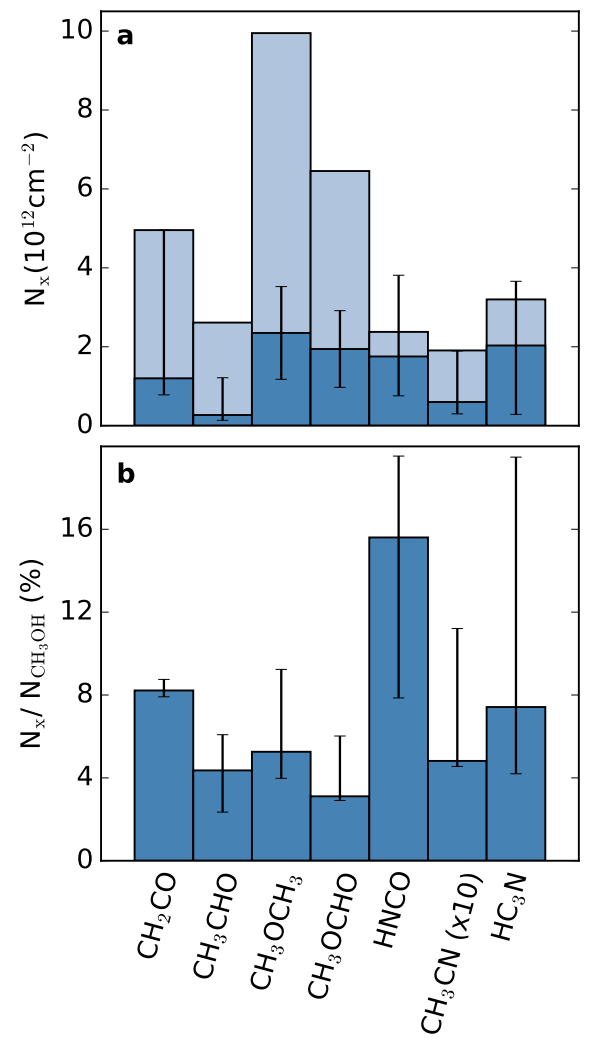

Figure 5. a: Median column densities of each molecule, calculated using detections only (light blue) and using survival analysis (dark blue). b: Abundances for each molecule calculated with survival analysis. In both panels, $\mathrm{CH}_{3} \mathrm{CN}$ has been scaled by 10 for clarity. Error bars span the first and third quartile determined using survival analysis.

sources with very high abundances and thus the statistics are biased, even with the inclusion of upper limits. $\mathrm{HC}_{3} \mathrm{~N}$ and $\mathrm{HNCO}$ also have fairly high column densities and are detected in 12 and 13 sources respectively, indicating that they are both common and abundant in low-mass protostellar environments.

Median abundances using survival analysis are shown in Figure 5b. Most molecules have median abundances of a few percent with respect to $\mathrm{CH}_{3} \mathrm{OH} ; \mathrm{CH}_{3} \mathrm{CN}$ is again the exception, with a median abundance an order of magnitude lower. The distribution of abundances, reflected in the error bars spanning the lower and upper quartile, is much tighter for oxygen-bearing molecules than for nitrogen-bearing molecules; this is discussed further in the following section.

\subsubsection{Column density and abundances distributions}

To explore the distributions of column densities before and after normalization to $\mathrm{CH}_{3} \mathrm{OH}$, Figure 6a shows histograms of each molecule's column density. For most molecules, the range of column densities spans an order of magnitude or more. The exceptions are $\mathrm{CH}_{3} \mathrm{OCH}_{3}$ and $\mathrm{CH}_{3} \mathrm{OCHO}$, for which only two detections of each molecule are available. The lower and upper quartiles for column densities are listed in Table $4 . \mathrm{HC}_{3} \mathrm{~N}$ has a particularly broad distribution, with over an order of magnitude difference between the lower and upper quartile values. $\mathrm{CH}_{2} \mathrm{CO}, \mathrm{CH}_{3} \mathrm{CHO}$, and $\mathrm{CH}_{3} \mathrm{CN}$ have fairly large spreads, with a factor of $\sim 6-7$ difference in lower and upper quartile values. The narrow distributions of $\mathrm{CH}_{3} \mathrm{OCH}_{3}$ and $\mathrm{CH}_{3} \mathrm{OCHO}$ are not informative due to the small number of detections.

When normalized to $\mathrm{CH}_{3} \mathrm{OH}$, the distribution of abundances of oxygen-bearing species tightens considerably as seen in the narrower distributions in Figure $6 \mathrm{~b}$ compared to $6 \mathrm{a}$, with most detections around $10 \%$ with respect to $\mathrm{CH}_{3} \mathrm{OH}$. In contrast, the $\mathrm{CH}_{3} \mathrm{OH}$-normalized abundance distributions for nitrogen-bearing species are broad. The lower and upper quartiles in Table 4 reflect this as well: for the abundances of oxygen-bearing species, upper quartile values are 1-2 times larger than lower quartile values. In contrast, upper quartile values for nitrogen-bearing species are 3-4 times larger than lower quartile values. Together, this suggests an oxygen chemistry that is connected to $\mathrm{CH}_{3} \mathrm{OH}$, whereas the nitrogen chemistry is regulated by other factors besides $\mathrm{CH}_{3} \mathrm{OH}$ abundances.

\subsection{COM Rotational temperatures}

For sources with at least 3 detections of a given molecule, rotational excitation temperatures could be derived using the rotational diagram method. These spanned from $4 \mathrm{~K}$ to $33 \mathrm{~K}$, with $\mathrm{CH}_{3} \mathrm{CHO}$ having the lowest temperature and $\mathrm{CH}_{3} \mathrm{CN}$ the highest. All rotational temperatures are quite low, indicating that the majority of emission does not come from a region that is both hot and dense. Both the temperature and density of protostars increase towards the center, therefore most emission must originate on larger scales. Since rotational temperatures depend on the excitation conditions and each molecule's excitation properties, they are typically not equal to the kinetic temperature. However, for molecules with similar dipole moments, rotational temperatures should be proportional to kinetic temperatures; for this reason we can compare $\mathrm{CH}_{3} \mathrm{CHO}$ and $\mathrm{CH}_{3} \mathrm{CN}$ with $\mathrm{CH}_{3} \mathrm{OH}$ to put qualitative constraints on their emission regions. As for previous observations of low-mass (Öberg et al. 2014) and high-mass (Bisschop et al. 2007; Fayolle et al. 2015) protostars, we find a relatively low rotational temperature for $\mathrm{CH}_{3} \mathrm{CHO}$, suggesting that it is mainly emitting from the cold outer envelope. Likewise, we find a higher rotational temperature for $\mathrm{CH}_{3} \mathrm{CN}$, suggesting that it emits from closer in.

The rotational temperatures of $\mathrm{CH}_{3} \mathrm{OCH}_{3}$ and $\mathrm{CH}_{3} \mathrm{OCHO}$ are both higher than the $\mathrm{CH}_{3} \mathrm{OH}$ rotational temperature in the two sources in which they are detected (B1-a and SVS 4-5). Given this, it is unlikely that 


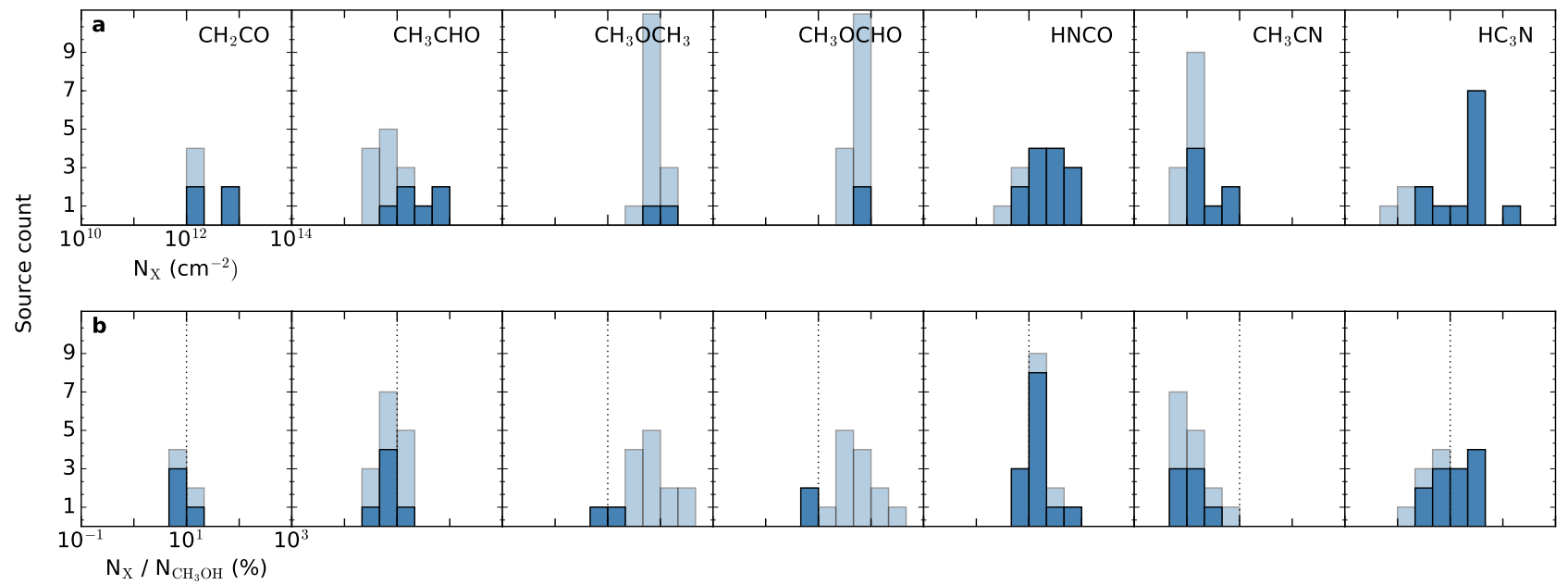

Figure 6. a: Histograms of observed column densities for each molecule. b: Abundances with respect to $\mathrm{CH}_{3} \mathrm{OH}$. The dotted black line represents 10\%. For both panels, detections are shown in dark blue, and upper limits in light blue.

the envelope is the sole origin of emission. This is consistent with observations of hot-core MYSOs in which both molecules are classified as hot molecules (Bisschop et al. 2007). We note, however, that $\mathrm{CH}_{3} \mathrm{OCH}_{3}$ and $\mathrm{CH}_{3} \mathrm{OCHO}$ have low signal-to-noise detections and are found only in two sources, and so further observations are required to confirm whether these molecules are indeed warm emitters in LYSOs.

\subsection{Correlation studies}

To explore chemical relationships between different COMs, we determined the correlations between each $\mathrm{COM}$ with one another and with $\mathrm{CH}_{3} \mathrm{OH}$. Strong positive correlations are expected for chemically related molecules (e.g. if one forms from another), or for molecules that depend similarly on an underlying variable such as envelope mass or temperature. When cor- relating column densities, some correlation is always expected since all molecules typically increase with an increasing total column density in a line of sight. This could in theory be divided out, but there are insufficient constraints on the physical characteristics of each source and on the origin of COM emission within each source to convert column densities to abundances with respect to $\mathrm{H}_{2}$. We therefore use the column density correlation strengths to infer which molecules are more closely related, with the caveat that some of the correlation may be due to source richness rather than chemical relatedness.

We calculate Pearson correlation coefficients for each COM with one another, shown in Table 5. Only detections are used for this calculation; because of this, only pairs of molecules which are detected in at least three of the same sources have correlation coefficients listed. Figure 7 shows scatterplots of each molecule's column density plotted against one another.

Table 5. Column density correlations between molecules.

\begin{tabular}{|c|c|c|c|c|c|}
\hline & $\mathrm{CH}_{3} \mathrm{OH}$ & $\mathrm{CH}_{2} \mathrm{CO}$ & $\mathrm{CH}_{3} \mathrm{CHO}$ & $\mathrm{CH}_{3} \mathrm{CN}$ & $\mathrm{HC}_{3} \mathrm{~N}$ \\
\hline $\mathrm{HNCO}$ & 0.69 [13] & $0.98[4]$ & $0.79[6]$ & $0.79[7]$ & $0.67[12]$ \\
\hline $\mathrm{HC}_{3} \mathrm{~N}$ & $0.78[12]$ & $0.57[3]$ & $0.82[6]$ & $0.71[7]$ & \\
\hline $\mathrm{CH}_{3} \mathrm{CN}$ & $0.90[7]$ & $0.99[3]$ & $0.96[5]$ & & \\
\hline $\mathrm{CH}_{3} \mathrm{CHO}$ & $0.90[6]$ & - & & & \\
\hline $\mathrm{CH}_{2} \mathrm{CO}$ & $0.99[4]$ & & & & \\
\hline
\end{tabular}

Note-Brackets indicate the number of sources with detections for both molecules. A dash indicates a pair of molecules with fewer than 3 sources in common. 


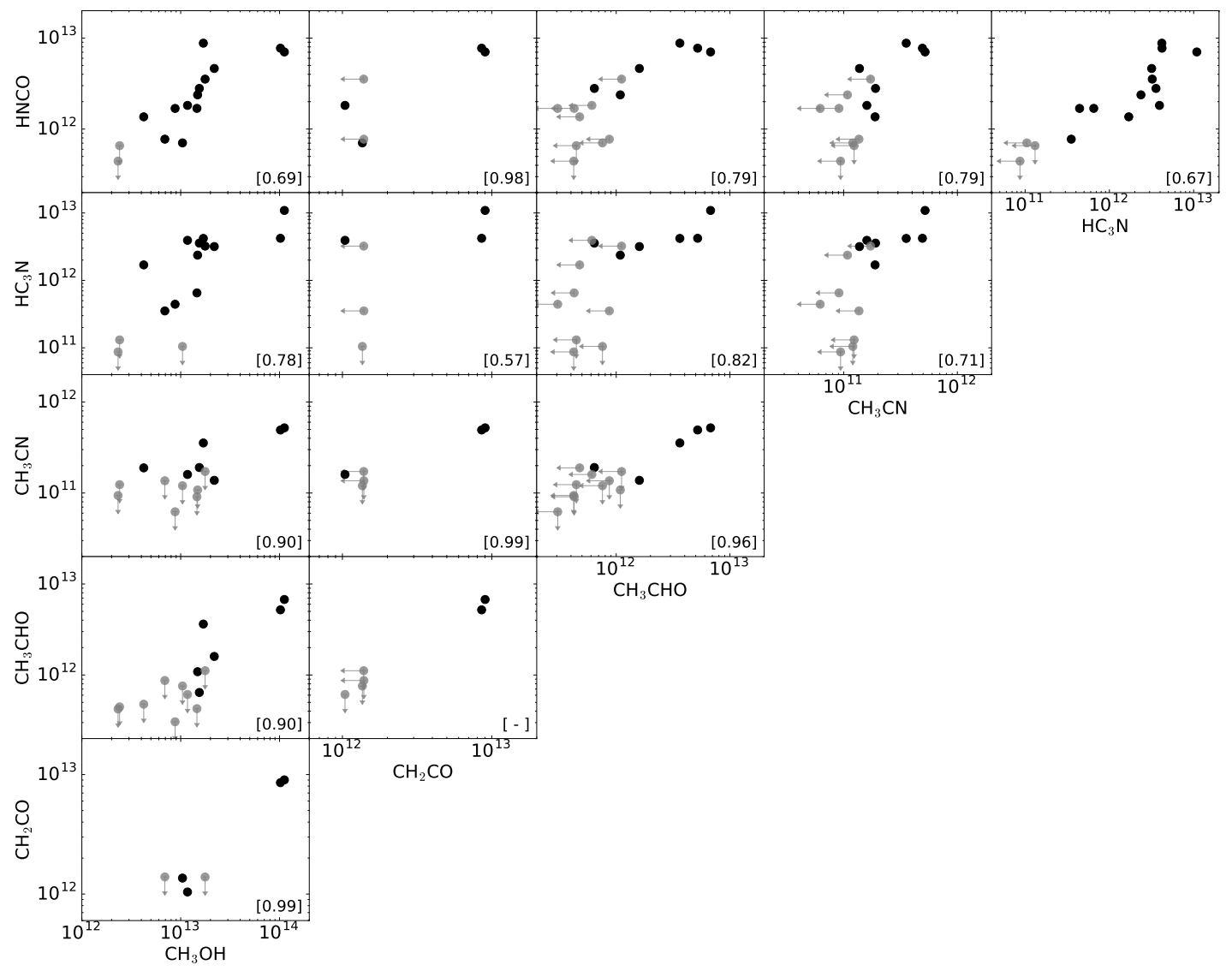

Figure 7. Column densities $\left(\mathrm{cm}^{-2}\right)$ of each molecule plotted against one another. Detections are shown as black circles and upper limits as grey arrows. Pearson correlation coefficients are listed in brackets.

and $\mathrm{HC}_{3} \mathrm{~N}$. The higher correlation of $\mathrm{CH}_{3} \mathrm{OH}$ with oxygen-bearing species compared to nitrogen-bearing species supports a scenario in which O-bearing COMs form from $\mathrm{CH}_{3} \mathrm{OH}$ processing. Within the nitrogenbearing family, there are no particularly strong relationships between any of the molecules. $\mathrm{HC}_{3} \mathrm{~N}$ is the least well-correlated with other molecules; this is unsurprising given that it is a carbon chain rather than a hydrogen-rich COM. $\mathrm{CH}_{3} \mathrm{OCH}_{3}$ and $\mathrm{CH}_{3} \mathrm{OCHO}$ correlations cannot be assessed since each is detected in only two sources.

We caution that the high correlation values for $\mathrm{CH}_{2} \mathrm{CO}$ with $\mathrm{CH}_{3} \mathrm{OH}$, $\mathrm{HNCO}$ and $\mathrm{CH}_{3} \mathrm{CN}$ may be an artifact of the low number of $\mathrm{CH}_{2} \mathrm{CO}$ detections: the $\mathrm{CH}_{2} \mathrm{CO}$ transition at $100.095 \mathrm{GHz}$ is only observable within the spectral range of the 6 objects in the pilot survey, of which it is detected in 4 . The lack of observational constraints for intermediate values of $\mathrm{CH}_{2} \mathrm{CO}$ hinders an interpretation of the true distribution of column densities.

Correlations of column densities with physical properties may also provide insight into the chemistry. To this end, we checked for correlations between envelope mass and bolometric luminosities (see Table 1) with our
COM column densities.

All molecules correlate positively with envelope mass (Figure 8). This is in part because more molecules are present in lines of sight containing more material. The correlation is strongest for $\mathrm{HC}_{3} \mathrm{~N}$ followed by $\mathrm{HNCO}$, with all other molecules showing weaker positive correlations. Among the nitrogen-bearing species, the observed sequence of correlation strengths with $\mathrm{M}_{\text {env }}$ are consistent with the expected origin of emission of each molecule. $\mathrm{HC}_{3} \mathrm{~N}$ is thought to form through gas-phase chemistry and emit from the cold envelope, and therefore should trace the envelope mass very well. HNCO is thought to emit from both the warm inner region and the cold envelope, which is consistent with a weaker correlation with envelope mass. Based on its rotational temperature, $\mathrm{CH}_{3} \mathrm{CN}$ is mainly present in the warmer, more compact region of the protostar, and should therefore not depend strongly on total envelope mass.

Interestingly, $\mathrm{CH}_{3} \mathrm{CHO}$ and $\mathrm{CH}_{2} \mathrm{CO}$ are also cold emitters, but unlike $\mathrm{HC}_{3} \mathrm{~N}$ they depend only weakly on envelope mass. This could be because these molecules form mainly on grain surfaces rather than via gas-phase chemistry.

Correlations with bolometric luminosity are also pos- 


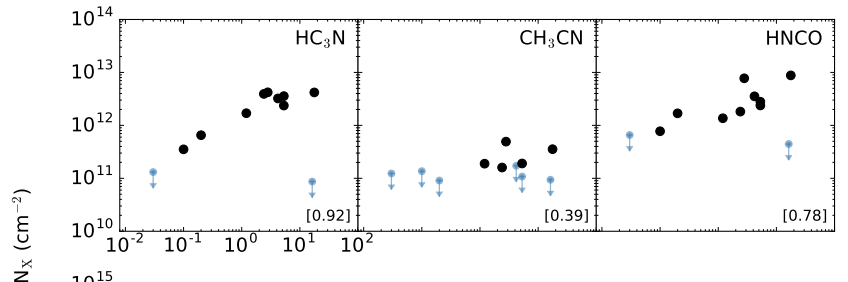

ż

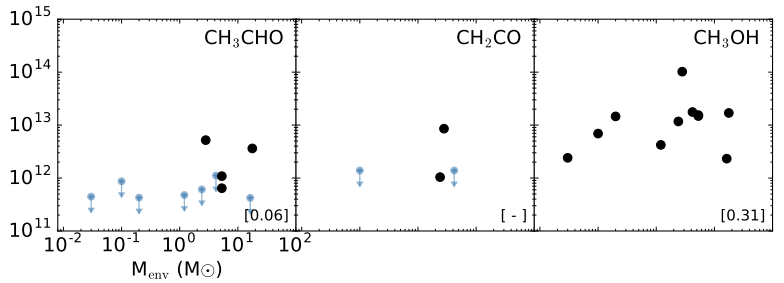

Figure 8. Column densities plotted against envelope mass. Upper limits due to molecule non-detections are shown in blue. Pearson correlation coefficients are shown in brackets in the lower right corner.

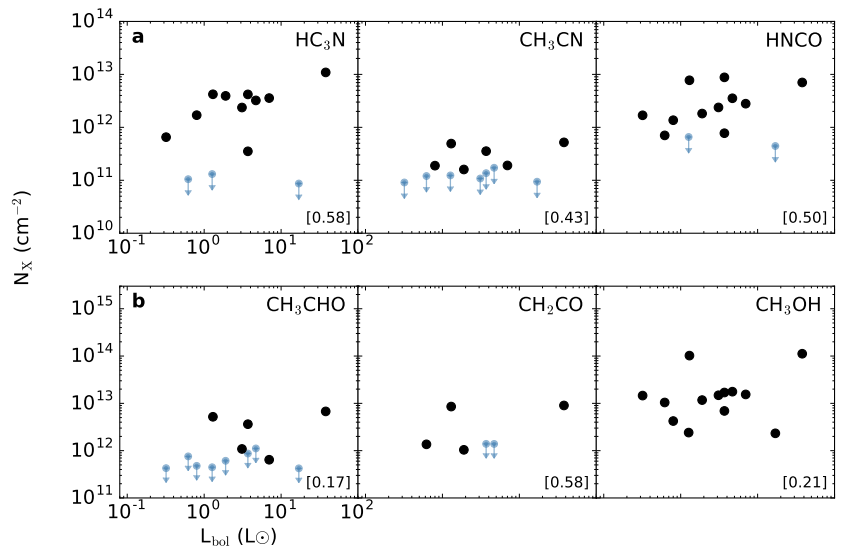

Figure 9. Column densities plotted against bolometric luminosity. Upper limits due to molecule non-detections. Pearson correlation coefficients are shown in brackets in the lower right corner.

itive, shown in Figure 9. In contrast to the envelope mass correlations, no single molecule stands out as particularly well-correlated with $\mathrm{L}_{\mathrm{bol}}$. This suggests that the weak correlations are not driven by chemistry, but rather excess excitation and ice desorption (affecting all molecules) around hotter protostars. The most obvious outlier in Figures 8 and 9 is L1448 IRS1, towards which no COMs are detected despite being one of the most massive and luminous of our sample. Massive sources are more likely to be young sources (e.g. Hatchell et al. 2007), so its unusual characteristics could be related to an early evolutionary stage.

All sources in the sample have been detected in water ice and in at least one of the minor ice constituents $\mathrm{NH}_{3}, \mathrm{CH}_{3} \mathrm{OH}$, and $\mathrm{CH}_{4}$ (see Table 1). In the context of $\mathrm{COM}$ formation, it is especially interesting to test whether the relative abundances of $\mathrm{O}$ - and N-bearing $\mathrm{COMs}$ are related to the relative ice abundances of $\mathrm{CH}_{3} \mathrm{OH}$ (the theoretical starting point of O-rich COM
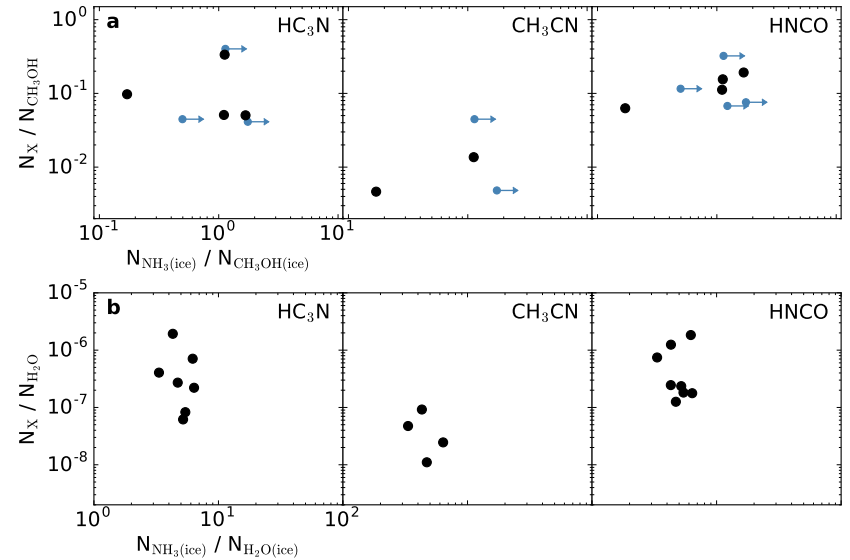

Figure 10. (a): Abundances with respect to $\mathrm{CH}_{3} \mathrm{OH}$ for the nitrogen-bearing species plotted against the observed $\mathrm{NH}_{3} / \mathrm{CH}_{3} \mathrm{OH}$ ice ratio. Lower limits due to non-detections of $\mathrm{CH}_{3} \mathrm{OH}$ ice are shown in blue. (b): Abundances with respect to $\mathrm{H}_{2} \mathrm{O}$ for the nitrogen-bearing species plotted against the observed $\mathrm{NH}_{3} / \mathrm{H}_{2} \mathrm{O}$ ice ratio.

chemistry) and $\mathrm{NH}_{3}$ (the main observed carrier of nitrogen in interstellar ices (Öberg et al. 2011a); $\mathrm{N}_{2}$ is also a possible reservoir of nitrogen in ices but cannot be observed directly). The number of sources with both $\mathrm{CH}_{3} \mathrm{OH}$ and $\mathrm{NH}_{3}$ detections is unfortunately small: only 10 sources have both detections, or one detection and one low upper limit, for $\mathrm{CH}_{3} \mathrm{OH}$ and $\mathrm{NH}_{3}$ ice columns. Of this, only a subset are rich in COMs, which limits the conclusiveness of any correlation studies. Figure 10 a shows the abundances with respect to $\mathrm{CH}_{3} \mathrm{OH}$ gas for the nitrogen-bearing species, plotted against the $\mathrm{NH}_{3} / \mathrm{CH}_{3} \mathrm{OH}$ ice ratio towards the same sources. $\mathrm{HC}_{3} \mathrm{~N} / \mathrm{CH}_{3} \mathrm{OH}$ gas-phase ratios are clearly not correlated with $\mathrm{NH}_{3} / \mathrm{CH}_{3} \mathrm{OH}$ ice ratios. Otherwise, there are no conclusive relationships between the abundance of $\mathrm{NH}_{3}$ ice and nitrogen-bearing molecules in this sample. At first glace, $\mathrm{CH}_{3} \mathrm{CN} / \mathrm{CH}_{3} \mathrm{OH}$ and $\mathrm{HNCO} / \mathrm{CH}_{3} \mathrm{OH}$ appear correlated with $\mathrm{NH}_{3} / \mathrm{CH}_{3} \mathrm{OH}$ ice ratios; however, the presence of lower limit outliers, one for $\mathrm{CH}_{3} \mathrm{CN}$ and two for HNCO, suggests that the complex cyanide chemistry may be only weakly dependent on $\mathrm{NH}_{3}$ ice abundances or altogether independent. Further ice observations may reveal these lower limits to be anomalous, or may reveal relationships between other nitrogen-bearing molecules and $\mathrm{NH}_{3}$ ice. Figure $10 \mathrm{~b}$ shows the abundances with respect to $\mathrm{H}_{2} \mathrm{O}$ instead of $\mathrm{CH}_{3} \mathrm{OH}$ for $\mathrm{NH}_{3}$ ice verses gas-phase nitrogen-bearing species. Again there is no obvious relationship, indicating that the lack of dependency of cyanide chemistry on $\mathrm{NH}_{3}$ is not an artifact of the normalizing species.

\section{CHEMICAL MODEL}

\subsection{Model description}


In order to compare our observational results for LYSO chemical abundances with current theoretical predictions, we have used the three-phase chemical kinetics code MAGICKAL (Garrod 2013) to simulate LYSO chemistry. The model version and the chemical network employed are those presented by Garrod et al. (2017); however, for the molecules of interest here, the differences between the work of Garrod (2013) and more recent implementions are very minor. The model considers both the gas-phase and grain-surface/ice-mantle formation and destruction of molecules. Complex organic molecules are largely assumed to have only dust grain-related formation mechanisms, in the absence of other information.

We run the model multiple times using a selection of physical conditions that are representative of values at a range of radial distances from the core center. We use results from this grid of chemical models to produce a composite, spatially-dependent picture of the chemistry in the LYSO.

We begin with a spherically symmetric physical model of a generic LYSO, with a power-law temperature profile adapted from Chandler \& Richer (2000):

$$
T(r)=60\left(\frac{r}{2 \times 10^{15} \mathrm{~m}}\right)^{-q}\left(\frac{L_{b o l}}{10^{5} L_{\odot}}\right)^{q / 2} \mathrm{~K},
$$

where we assume $q=2 / 5$ and $L_{b o l}$ is either 1 or $10 \mathrm{~L}_{\odot}$. For the density profile, we assume the power law:

$$
n_{H}(r)=n_{1000 A U}\left(\frac{r}{1000 A U}\right)^{-\alpha} .
$$

Based on the median values determined from radiative transfer modeling of low-mass protostars in Jørgensen et al. (2002), we assume $n_{1000 A U}=10^{6} \mathrm{~cm}^{-3}$ and $\alpha=$ 1.5. We also assume an inner radius where $\mathrm{T}=250 \mathrm{~K}$, again following Jørgensen et al. (2002), and an outer radius where $\mathrm{T}=10 \mathrm{~K}$. In the $L_{b o l}=10 \mathrm{~L}_{\odot}$ case, a temperature of $250 \mathrm{~K}$ is achieved at a radius of $3.8 \mathrm{AU}$, with a corresponding density of $4.27 \times 10^{9} \mathrm{~cm}^{-3}$ at that position. In the $L_{b o l}=1 \mathrm{~L}_{\odot}$ case, the peak temperature of $250 \mathrm{~K}$ corresponds to a radius of $1.2 \mathrm{AU}$, at which a density of $2.89 \times 10^{10} \mathrm{~cm}^{-3}$ is achieved. The latter value is taken as the maximum density for which chemicalmodel data are required (in either luminosity case).

To populate the radial density profile with chemical data, we determine a total of 51 densities for which models are to be run, ranging logarithmically from the maximum of $2.89 \times 10^{10} \mathrm{~cm}^{-3}$ down to $3.45 \times 10^{4} \mathrm{~cm}^{-3}$. For each of these densities, we run a dedicated hot-core type model, using a two-stage approach (following Garrod 2013): stage one consists of an isothermal collapse from density $3000 \mathrm{~cm}^{-3}$ up to the final density chosen for that run; in stage two, this density is fixed and the gas and dust temperatures gradually rise to a maximum of $400 \mathrm{~K}$, using the "intermediate" warm-up timescale (which goes from $8-200 \mathrm{~K}$ in $2 \times 10^{5} \mathrm{yr}$, producing a total warm-up timescale from $8-400 \mathrm{~K}$ of $2.85 \times 10^{5} \mathrm{yr}$ ).

For either the $L_{b o l}=1 \mathrm{~L}_{\odot}$ or $10 \mathrm{~L}_{\odot}$ case, models with densities that fall within the maximum and miminum values are chosen and placed at the appropriate radius. Then, from within each model, the abundances of all simulated molecules are extracted according to the instantaneous temperature achieved during warm-up, as determined by the temperature profile at that specific radius. To obtain data at the precise temperatures required, the model output data are interpolated between output temperature values; the temperature resolution obtained in the models is always less than $1 \%$ of the absolute value.

In this way, each model placed at a specific position in the density profile is fixed in time according to the local temperature. To account for uncertainties in the temperature profile, we also extract chemical profiles corresponding to local temperature values that are $10 \%$ lower and $10 \%$ higher temperatures than those given by the fiducial temperature profile in equation 4.

The resulting radial profiles for all molecules are then used to calculate the line-of-sight column densities that would be observed with a single-dish telescope. To do so, we convolve the molecular abundance $\frac{n_{X}}{n_{H}}(r)$, hydrogen density $n_{H}(r)$, and emitting area $A(r)$ at each radius. We then integrate this from our minimum to maximum radii to yield the total number of molecules of a given species within the beam:

$$
n_{X}=\int_{r_{\min }}^{r_{\max }} A(r) n_{H}(r) \frac{n_{X}}{n_{H}}(r) .
$$

The column density is then simply:

$$
N_{X}=\frac{n_{X}}{\pi r_{b}^{2}},
$$

where $r_{b}$ is the beam radius. We assume a 10 " beam radius based on the size of the observing beam (Section 2), and a distance to the model protostar of 200pc. In order to account for the cylindrical line of sight, we express $A(r)$ as:

$$
A(r)= \begin{cases}4 \pi r^{2}, & \text { if } r \leq r_{b} \\ 2 \pi\left[r_{b}^{2}+\left(r-\sqrt{r^{2}-r_{b}^{2}}\right)^{2}\right], & \text { if } r>r_{b}\end{cases}
$$

In other words, while the radius is smaller than the beam radius, the emitting surface corresponds to the surface area of a sphere at that radius. Beyond the beam radius, the emitting surface consists of spherical caps in front of and behind a sphere of radius $=r_{b}$. These caps have a constant fixed base radius $r_{b}$ and a height that varies depending on the radius. Figure 11 illustrates how this 


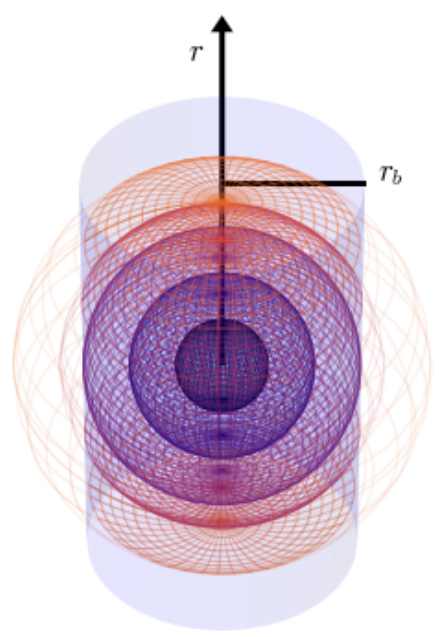

Figure 11. Schematic of emitting surfaces contained within a cylindrical beam for different radial shells.

treatment accounts for material in the line of sight while excluding material outside the beam.

Our use of a single warm-up timescale keeps the model grid to a manageable size. The choice to use the "intermediate" timescale is based on its ability to best reproduce the abundances of hot-core type molecules at the high temperatures of such sources. In the case of the low-mass protostars that concern this work, the "fast" timescale could also be an appropriate choice; however, the differences between the "fast" and "intermediate" results are mainly quantitative rather than qualitative (in contrast with the "slow" warm-up timescale models). Differences between peak abundances calculated by these models are likely to be on the order of a factor of a few. Such variations are acceptable in the context of our generic model, but the modeling of specific sources in the future may be tailored to more specific warm-up timescales, using an explicitly determined infall speed.

\subsection{Model results}

Figure 12 shows the 1D radial profiles of COM fractional abundances for both the $1 \mathrm{~L}_{\odot}$ and $10 \mathrm{~L}_{\odot}$ protostar simulations. The variations in temperature by $\pm 10 \%$ do not produce significant changes in the peak abundances; rather, they alter the radial onset of peak values, thereby changing the calculated column densities. Generally, trends in the radial abundances are consistent with our expectations based on observations: $\mathrm{CH}_{3} \mathrm{OCH}_{3}$ and $\mathrm{CH}_{3} \mathrm{OCHO}$, which are thought to emit from warm inner regions of the protostar, drop off in abundance moving to larger radii, while the "cold" emitters $\mathrm{CH}_{2} \mathrm{CO}, \mathrm{CH}_{3} \mathrm{CHO}, \mathrm{HC}_{3} \mathrm{~N}$, and $\mathrm{HNCO}$ maintain a high abundance at all radii. Interestingly, $\mathrm{CH}_{3} \mathrm{CN}$ has a profile more similar to the cold emitters despite its observationally derived warmer rotational temperature. We discuss the chemistry of these models further in Sec- tion 5.2.

\section{DISCUSSION}

\subsection{COM formation chemistry}

Correlations among COMs and between COMs and source properties provide constraints on their chemistry. In this subsection, we discuss the formation chemistry of individual molecules based on their rotational temperatures and observed correlations with $\mathrm{CH}_{3} \mathrm{OH}$, protostellar properties, and ice abundances (Figures 7-10).

$\mathrm{CH}_{2} \mathrm{CO}: \mathrm{CH}_{2} \mathrm{CO}$ formation has been demonstrated experimentally by ice processing, with proposed formation mechanisms of either $\mathrm{C}_{2} \mathrm{O}$ hydrogenation (Maity et al. 2014) or vinyl alcohol formation and decomposition (Hudson \& Loeffler 2013). However, theory indicates that $\mathrm{H}$ addition to $\mathrm{C}_{2} \mathrm{O}$ is the dominant pathway (Garrod et al. 2008). The strong correlation of $\mathrm{CH}_{2} \mathrm{CO}$ with $\mathrm{CH}_{3} \mathrm{OH}$ along with its low rotational temperature suggest that ketene forms via atom addition reactions in simple ices (i.e. $\mathrm{CO}+\mathrm{C} \rightarrow \mathrm{C}_{2} \mathrm{O}$, followed by hydrogenation to $\mathrm{CH}_{2} \mathrm{CO}$ ) similar to $\mathrm{CH}_{3} \mathrm{OH}$ formation.

$\mathrm{CH}_{3} \mathrm{CHO}: \mathrm{CH}_{3} \mathrm{CHO}$ also correlates strongly with $\mathrm{CH}_{3} \mathrm{OH}$. $\mathrm{CH}_{3} \mathrm{CHO}$ has a very low excitation temperature $(8 \mathrm{~K})$ and is therefore likely a zeroth or first-generation COM. As mentioned in Section 3.5, $\mathrm{CH}_{3} \mathrm{CHO}$ does not correlate well with $\mathrm{M}_{\mathrm{env}}$; this is in contrast to the gas-phase chemistry product $\mathrm{HC}_{3} \mathrm{~N}$. $\mathrm{CH}_{3} \mathrm{CHO}$ is therefore not likely to be a gas-phase product. Both experiments (Bennett et al. 2005; Öberg et al. 2009) and modeling (Garrod et al. 2008) demonstrate efficient formation of acetaldehyde from radical products of $\mathrm{CH}_{3} \mathrm{OH}$, namely $\mathrm{CH}_{3}$ and $\mathrm{HCO} . \mathrm{CH}_{3} \mathrm{OH}$ processing is not the only pathway to $\mathrm{CH}_{3} \mathrm{CHO}\left(\mathrm{CH}_{3}\right.$ can also be derived from the dissociation of $\mathrm{CH}_{4}$, and the $\mathrm{HCO}$ radical can form from $\mathrm{CO}$ hydrogenation or dissociation of $\mathrm{H}_{2} \mathrm{CO}$ ), but the tight correlation suggests that $\mathrm{CH}_{3} \mathrm{OH}$ is an important precursor to $\mathrm{CH}_{3} \mathrm{CHO}$

$\mathrm{CH}_{3} \mathrm{OCH}_{3}$ and $\mathrm{CH}_{3} \mathrm{OCHO}$ : Methyl formate and dimethyl ether have fairly warm rotational temperatures in our survey, with averages of $17 \mathrm{~K}$ and $16 \mathrm{~K}$, respectively. This is much warmer than $\mathrm{CH}_{3} \mathrm{CHO}$, suggesting that these molecules originate closer to the central protostar. Both molecules are thought to form from recombinations of radical products of methanol, and our data is consistent with this formation scenario: $\mathrm{CH}_{3} \mathrm{OCH}_{3}$ and $\mathrm{CH}_{3} \mathrm{OCHO}$ are the most abundant towards sources with high $\mathrm{CH}_{3} \mathrm{OH}$ column densities. However, with only two detections this cannot be ascertained with any confidence. Firm detections in sources with lower column densities will help to elucidate this relationship. We note that this will likely require ALMA observations, as the observations presented here are already quite deep. Spatially resolved observations towards the sources with 


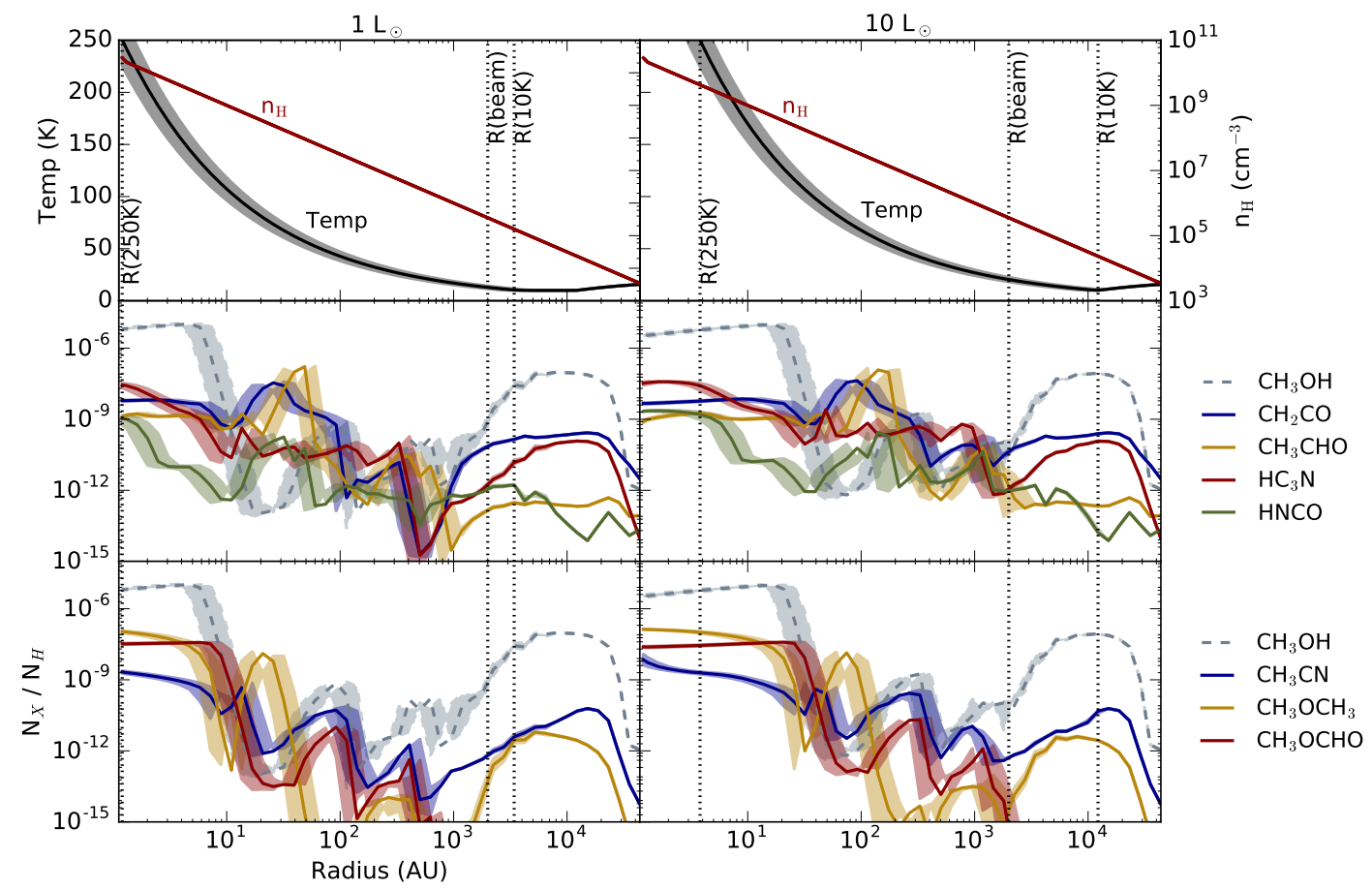

Figure 12. Chemical models for $1 \mathrm{~L}_{\odot}$ and $10 \mathrm{~L} \odot$ LYSO simulations. In all panels, dotted vertical lines mark the inner radius at $250 \mathrm{~K}$, the beam radius of 10 " for an object at $200 \mathrm{pc}$, and the outer radius for a $10 \mathrm{~K}$ cutoff temperature. The shaded regions around each line represent results from temperature profiles 10\% higher and 10\% lower than the fiducial run. Top panels: temperature and density profiles (equations 4 and 5). Middle panels: fractional abundances of the typically "cold" emitters $\mathrm{CH}_{2} \mathrm{CO}, \mathrm{CH}_{3} \mathrm{CHO}, \mathrm{HC}_{3} \mathrm{~N}$, and $\mathrm{HNCO}$. Bottom panels: abundances of the typically "warm" emitters $\mathrm{CH}_{3} \mathrm{CN} \mathrm{CH}_{3} \mathrm{OCH}_{3}$, and $\mathrm{CH}_{3} \mathrm{OCHO}$. $\mathrm{CH}_{3} \mathrm{OH}$ is shown for comparison in both the middle and bottom panels.

detections would also be helpful in constraining the formation chemistry.

HNCO: The chemical origin of HNCO is still not particularly well understood. Recent experiments have shown that $\mathrm{HNCO}$ can be produced in ices from $\mathrm{CO}$ reacting with $\mathrm{NH}$ radicals before $\mathrm{N}$ is fully hydrogenated to $\mathrm{NH}_{3}$ (Fedoseev et al. 2015), or from simultaneous $\mathrm{UV}$ irradiation and hydrogenation of $\mathrm{NO}$ in $\mathrm{CO}, \mathrm{H}_{2} \mathrm{CO}$, and $\mathrm{CH}_{3} \mathrm{OH}$ ices (Fedoseev et al. 2016). Modeling by Garrod et al. (2008) shows that HNCO formation is efficient only by the gas-phase destruction of more complex grain-surface products, urea in particular. However, the recent model of Belloche et al. (2017) suggests reaction between $\mathrm{NH}$ and $\mathrm{CO}$ may be efficient (see Section 5.2). Several aspects of our HNCO observations support a scenario in which HNCO mainly forms through atom addition ice chemistry in the cold envelope. First, the ubiquity of $\mathrm{HNCO}$ in our sources favors an early formation (i.e. zeroth-generation $\mathrm{COM}$ chemistry): its detection towards sources with no other detections precludes a formation chemistry dependent on high gas-phase abundances of large complex molecules (i.e. second-generation formation). Moreover, HNCO correlates relatively well with envelope mass, indicative of formation in the envelope. Finally, HNCO column densities are fairly well correlated with $\mathrm{CH}_{3} \mathrm{OH}$ for sources with low column densities of $\mathrm{CH}_{3} \mathrm{OH}$, but the two sources with much higher $\mathrm{CH}_{3} \mathrm{OH}$ column densities (B1-a and SVS 4-5) do not display a comparable increase in HNCO column density. This is consistent with a scenario of coformation of $\mathrm{HNCO}$ and $\mathrm{CH}_{3} \mathrm{OH}$ in the cold envelope, while $\mathrm{HNCO}$ destruction in the core region could explain the saturation behavior.

$\mathrm{CH}_{3} \mathrm{CN}: \mathrm{CH}_{3} \mathrm{CN}$ has the highest rotational temperature of all the COMs observed, with an average of $27 \mathrm{~K}$; together with the lack of correlation between $\mathrm{CH}_{3} \mathrm{CN}$ and $\mathrm{M}_{\mathrm{env}}$, this suggests a more centrally concentrated emission origin and an efficient "lukewarm" pathway to $\mathrm{CH}_{3} \mathrm{CN}$. In hot cores, $\mathrm{CH}_{3} \mathrm{CN}$ is thought to form mainly on grain surfaces through recombination of $\mathrm{CH}_{3}+\mathrm{CN}$, followed by sublimation above $\sim 90 \mathrm{~K}$ (Garrod et al. 2008). A gas-phase channel, involving $\mathrm{HCN}+\mathrm{CH}_{3}^{+} \rightarrow$ $\mathrm{CH}_{3} \mathrm{CNH}^{+}$followed by $\mathrm{CH}_{3} \mathrm{CNH}^{+}+\mathrm{e}^{-} \rightarrow \mathrm{CH}_{3} \mathrm{CN}+$ $\mathrm{H}$, may become important at lower temperatures. In our sample, $\mathrm{CH}_{3} \mathrm{CN}$ correlates well with $\mathrm{CH}_{3} \mathrm{OH}$, indicating that these molecules have similar underlying chemistries and therefore that ice chemistry may be important in $\mathrm{CH}_{3} \mathrm{CN}$ formation. However, as discussed in Section 5.2, the chemical model relies on gas-phase chemistry to efficiently form $\mathrm{CH}_{3} \mathrm{CN}$ at these temperatures. Thus, there is some tension between the model and observations in inferring the dominant $\mathrm{CH}_{3} \mathrm{CN}$ formation channel. Fi- 
nally, $\mathrm{CH}_{3} \mathrm{CN}$ has no obvious correlation with the $\mathrm{NH}_{3}$ ice column, indicating that cyanide production in the ice is not linked to $\mathrm{NH}_{3}$.

$H_{3} N$ : $\mathrm{HC}_{3} \mathrm{~N}$ has the most scatter of any molecule in its correlation with $\mathrm{CH}_{3} \mathrm{OH}$. This is unsurprising given that cyanopolyyne observations can be well-reproduced by purely gas-phase models (Herbst \& van Dishoeck 2009); any correlation with $\mathrm{CH}_{3} \mathrm{OH}$ is likely related to the scale of the object and not a reliance on $\mathrm{CH}_{3} \mathrm{OH}$ chemistry. This is also consistent with the weak correlation of $\mathrm{HC}_{3} \mathrm{~N}$ with all other COMs, discussed in Section 3.5. The tight correlation between envelope mass and $\mathrm{HC}_{3} \mathrm{~N}$ abundance and the obvious lack of correlation with $\mathrm{NH}_{3}$ ice further support this. These observations support the general notion that hydrocarbon species are mainly gas-phase products, and do not require grainsurface chemistry to explain their abundances. Interestingly, Graninger et al. (2016) found a positive correlation between the carbon chain $\mathrm{C}_{4} \mathrm{H}$ and $\mathrm{CH}_{3} \mathrm{OH}$ in these LYSOs; this suggests that nitrogen-bearing carbon chains have distinct gas-phase formation chemistries from unsubstituted carbon chains.

\subsection{Modeled vs. observed chemistry}

Figure 13a compares beam-averaged COM abundances with respect to $\mathrm{CH}_{3} \mathrm{OH}$ for our observations and modeling results (Section 4). For the observations, median values are shown as pink stars, with error bars spanning the first and third quartile. For the model, we show results from both $1 \mathrm{~L}_{\odot}$ (light blue) and $10 \mathrm{~L}_{\odot}$ (dark blue) temperature profiles. Triangles represents results from the fiducial temperature profiles, and error bars show the results for temperature profiles $10 \%$ higher and $10 \%$ lower.

Apart from $\mathrm{HNCO}$, the model abundances are within an order of magnitude of the observations. Agreement within an order of magnitude is very reasonable given modeling uncertainties in the reaction rates as well as observational uncertainties in the different excitation properties of the molecules being compared. In particular, the systematic underestimation of most molecules by the model may be due in part to an excess of $\mathrm{CH}_{3} \mathrm{OH}$ in the model at large radii, caused by overactive chemical desorption of methanol at cold temperatures. The underproduction of HNCO by several orders of magnitude is likely a result of an over-estimated barrier for the grain surface process of $\mathrm{NH}+\mathrm{CO} \rightarrow \mathrm{HNCO}$, which impedes efficient grain-surface formation of $\mathrm{HNCO}$ in the model. While there are no strong constraints on this barrier from laboratory work, recent modeling work by Belloche et al. (2017) tested several values ranging from $1000-2500 \mathrm{~K}$, the latter value being the one used in our present study. They found that the barrier is required to be no greater than $\sim 1500 \mathrm{~K}$ in order to produce sufficient

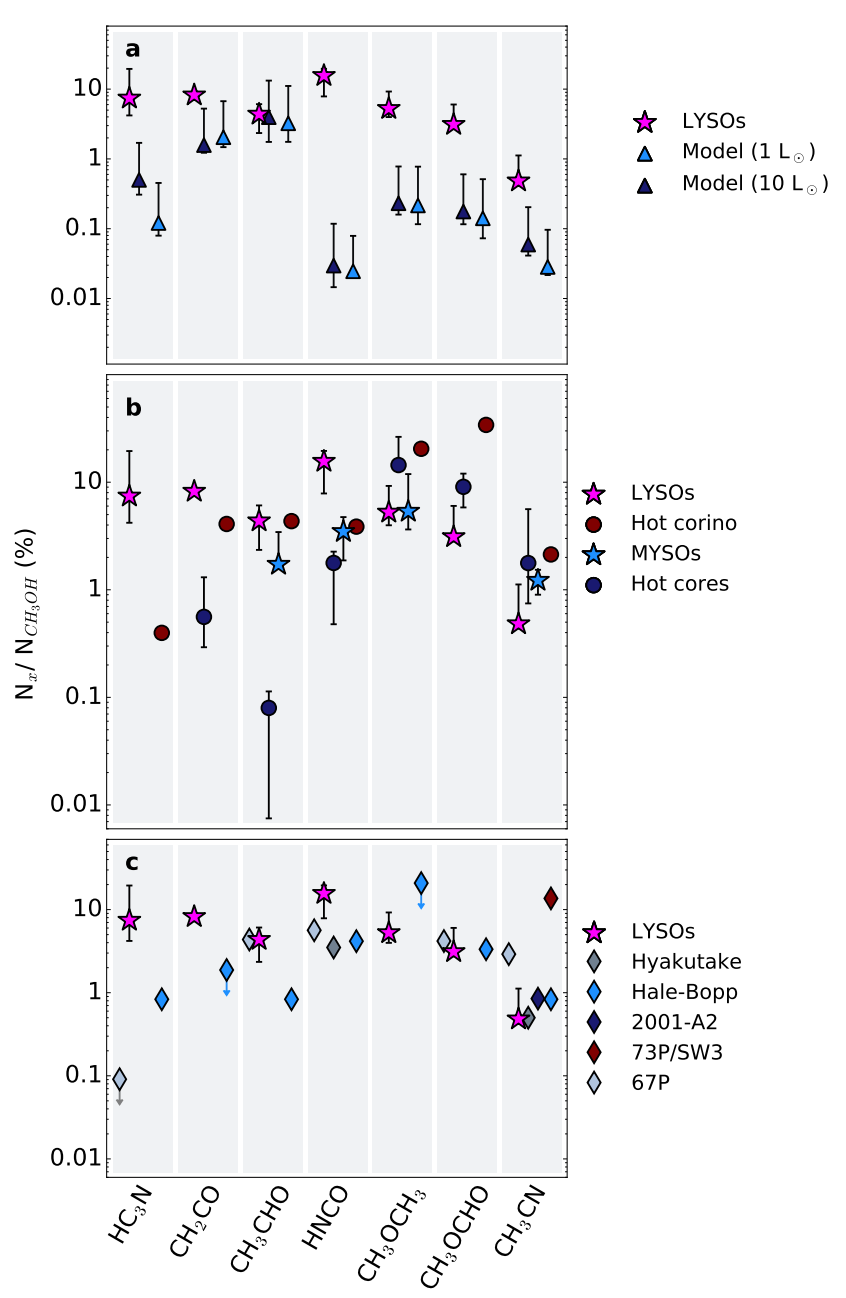

Figure 13. COM abundances with respect to $\mathrm{CH}_{3} \mathrm{OH}$ across different types of objects. For all panels, LYSOs are shown as pink stars. Scatterpoints represent median values and error bars span the first and third quartile. a: Comparison with abundances from the protostellar warm-up model described in Section 4 for $1 \mathrm{~L}_{\odot}$ (light blue) and $10 \mathrm{~L}_{\odot}$ (dark blue) profiles. Error bars span the results for temperature profiles $10 \%$ higher and $10 \%$ lower than the fiducial run. b: Comparison of different star formation environments. Hot corinos (van Dishoeck et al. 1995; Cazaux et al. 2003) are shown in dark pink; MYSOs (Fayolle et al. 2015) in light blue; and hot cores (Bisschop et al. 2007) in dark blue. c: Comparison with observations of solar system comets (Crovisier et al. 2004; Mumma \& Charnley 2011; Goesmann et al. 2015).

$\mathrm{HNCO}$ to explain observed abundances of $\mathrm{CH}_{3} \mathrm{NCO}$. The value of around $2500 \mathrm{~K}$ used in the present model was adopted by Garrod et al. (2008), when this reaction was first introduced into the network, and was based on the barrier found for the analogous reaction $\mathrm{H}+\mathrm{CO} \rightarrow$ $\mathrm{HCO}$. Reducing the current value of $2500 \mathrm{~K}$ to the $1500 \mathrm{~K}$ recommended by Belloche et al. may therefore improve agreement with our observational data in future model iterations.

Figure 14 shows the fraction of each molecule's total column density coming from different temperature 


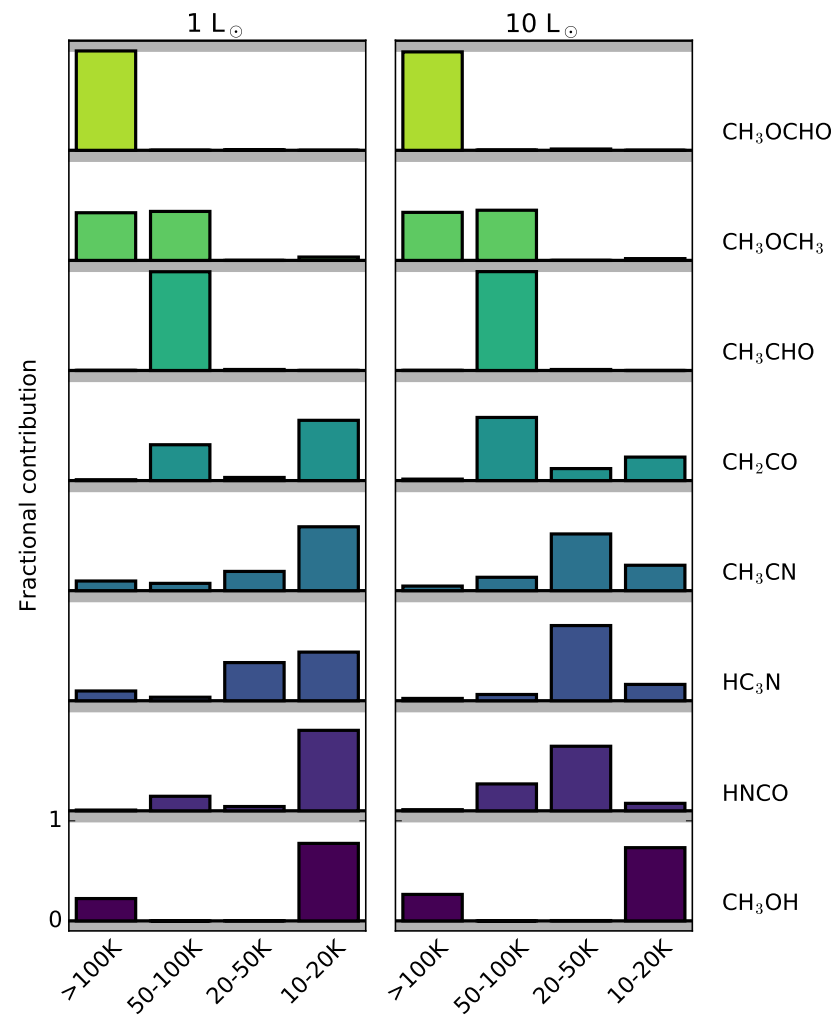

Figure 14. Model results showing the fraction of each molecule's total column density that comes from different temperature ranges. For each molecule, the y scale is the same as is indicated for $\mathrm{CH}_{3} \mathrm{OH}$.

ranges in the model, which can be compared with measured rotational temperatures. We note that if the observed line transitions are not in LTE then the measured rotational temperatures will underestimate the kinetic temperature. To assess how much this impacts our observations, we run RADEX models for molecules with both collisional constants and rotational temperatures $\left(\mathrm{HC}_{3} \mathrm{~N}, \mathrm{CH}_{3} \mathrm{CN}\right.$, and $\mathrm{CH}_{3} \mathrm{OH}$; van der Tak et al. 2007) using the measured rotational temperatures and column densities as inputs and assuming the density and temperature profiles in Section 4.1. We find that $\mathrm{CH}_{3} \mathrm{CN}$ and $\mathrm{HC}_{3} \mathrm{~N}$ observations are well reproduced by the simulated RADEX emission while $\mathrm{CH}_{3} \mathrm{OH}$ is not. Therefore, for some molecules (especially oxygen-bearing species), the measured rotational temperatures and column densities may be affected by non-LTE effects.

We also estimate the temperature above which $\mathrm{HC}_{3} \mathrm{~N}$, $\mathrm{CH}_{3} \mathrm{CN}$, and $\mathrm{CH}_{3} \mathrm{OH}$ should be in LTE. This is done using the collisional constants for these molecules (Schöier et al. 2005), again assuming the model density and temperature profiles. We find that most lines transition to non-LTE conditions at densities corresponding to 1025K. Most molecules have observed rotational temperatures around or below this range; this suggests that at least some component of our observed emission is sub- thermal, consistent with the RADEX results. Therefore, in comparing with the model emission temperatures, we emphasize that the measured rotational temperatures should be regarded as broadly corresponding to inner, outer, and intermediate emission origins, and likely do not correspond exactly to the actual kinetic temperature.

$\mathrm{CH}_{3} \mathrm{OCHO}$ and $\mathrm{CH}_{3} \mathrm{OCH}_{3}$ are both found at warm to hot temperatures $(>50 \mathrm{~K})$ in the model, compared with rotational temperatures of $16 \mathrm{~K}$ and $17 \mathrm{~K}$, respectively. However, these molecules are detected towards only 2 sources, and only in a few detected lines, so it is difficult to assess how serious these constraints are. They seem to rule out, however, that the emission is coming exclusively from the hot material as predicted by the model. $\mathrm{HC}_{3} \mathrm{~N}, \mathrm{HNCO}, \mathrm{CH}_{2} \mathrm{CO}$, and $\mathrm{CH}_{3} \mathrm{CN}$ are all dominated by temperatures colder than $50 \mathrm{~K}$ in the model. The available rotational temperatures (14K for $\mathrm{HC}_{3} \mathrm{~N}$ and $27 \mathrm{~K}$ for $\mathrm{CH}_{3} \mathrm{CN}$ ) are fully consistent with this. Thus, the model successfully reproduces the envelope-dominated, low-temperature chemistry seen in these LYSOs, without the need for hot-core chemistry. This is true even for $\mathrm{CH}_{3} \mathrm{CN}$, which is typically considered a hot core molecule. The gas-phase pathway described in Section 5.1 is mainly responsible for this lukewarm formation in the model, and is driven by an enhancement of gas-phase HCN. This in turn is due to either sublimation of HCN from grains in warmer regimes $(\sim 40 \mathrm{~K})$, or purely gas-phase HCN formation in cooler regimes. The observed rotational temperature of $27 \mathrm{~K}$ is consistent with some combination of low and intermediate temperature production of $\mathrm{CH}_{3} \mathrm{CN}$.

$\mathrm{CH}_{3} \mathrm{CHO}$ is found entirely at warm temperatures (50$100 \mathrm{~K}$ ) in the model due to desorption of $\mathrm{CH}_{3} \mathrm{CHO}$ formed on grain surfaces; this is much higher than its observed $8 \mathrm{~K}$ rotational temperature. Unlike methyl formate and dimethyl ether, acetaldehyde has very wellpopulated rotational diagrams and therefore the rotational temperature is more secure. It seems, then, that the observed $\mathrm{CH}_{3} \mathrm{CHO}$ is being produced through some cold formation pathway other than the radical recombination chemistry included in the model.

The majority of $\mathrm{CH}_{3} \mathrm{OH}$ in the model is found at cold temperatures (10-20K). Cold $\mathrm{CH}_{3} \mathrm{OH}$ in the model is likely somewhat over-abundant due to an overactive chemical desorption, contributing to the systematic under-estimation of other COM abundances seen in Figure 13. Even so, observational rotational temperatures for $\mathrm{CH}_{3} \mathrm{OH}$ in these sources are $\sim 5-6 \mathrm{~K}$ (Graninger et al. 2016), consistent with a mainly cold origin. This suggests that while the magnitude of cold $\mathrm{CH}_{3} \mathrm{OH}$ in the model may be too high, the trend of dominantly cold emission is appropriate.

The high efficiency of cold chemical desorption of 
methanol is a result of the rapid abstraction of hydrogen from methanol by $\mathrm{H}$ atoms, followed by rehydrogenation by another $\mathrm{H}$ atom. Chemical desorption occurs in $0.24 \%$ of re-hydrogenation cases, as determined by the method of Garrod et al. (2007), using an efficiency factor $a=0.01$. The abstraction and rehydrogenation of methanol on the grains in this model is sufficiently fast to produce an excess of gas-phase methanol, while the total remaining on the grains is affected only to a relatively small degree. The CO$\mathrm{H}_{2} \mathrm{CO}-\mathrm{CH}_{3} \mathrm{OH}$ system on grains is complex, with reactions that allow inter-conversion in both directions. The barriers and branching ratios for many of these processes are poorly-defined. The return of grain surface-formed methanol to the gas phase is therefore a function of both its formation/re-formation on grains and the efficiency of desorption, which is also poorly constrained.

In addition to gas-phase abundances, the model also provides ice abundances. In practice, observations of ices are made in absorbance and therefore trace a pencil beam of protostellar material rather than the entire envelope. To compare with observed ice column densities, therefore, the number density of each ice species in the model is simply integrated out to the maximum radius. The model produces fairly consistent values with the measured ice columns in Table 1 , with $\mathrm{H}_{2} \mathrm{O}$ column densities on the order $10^{19} \mathrm{~cm}^{-2}$ compared with observed values of $10^{18}-10^{19} \mathrm{~cm}^{-2}$. $\mathrm{CH}_{3} \mathrm{OH}$ model ice abundances with respect to water are around $7 \%$, which is consistent within a factor of a few of measured values, while $\mathrm{NH}_{3}$ model abundances of $19 \%$ are somewhat high compared to observations.

In the model, the number densities of ice species peak just outside of the ice sublimation line around $130 \mathrm{~K}$, corresponding to $6 \mathrm{AU}$ in the $1 \mathrm{~L}_{\odot}$ case and $21 \mathrm{AU}$ in the $10 \mathrm{~L}_{\odot}$ case. This is a much smaller spatial scale than our gas-phase observations, and therefore ice absorption measurements are likely not probing the same radius as the gas-phase observations. Nonetheless, observed gas and ice abundances should still be related for molecules that form primarily in the ice phase, assuming that ice compositions do not evolve substantially between the outer and inner envelope. Indeed, in the model the ice abundance profiles are essentially flat from the $10 \mathrm{~K}$ radius in to the sublimation line. Ices observed in absorption close to the sublimation line should therefore preserve a similar composition to ices in the outer envelope, from which many of the observed gas-phase COMs likely originate.

\subsection{Massive vs. low-mass protostellar chemistry}

We next compare observations of COMs towards different types of protostars. We consider our sample of low-mass protostars without clear evidence for hot cori- nos (subsequently termed LYSOs), the hot corino source IRAS 16293-2422, and analogously high-mass protostars without and with hot cores (termed MYSOs and hot cores, respectively). Abundance measurements for the hot corino are taken from van Dishoeck et al. (1995) and Cazaux et al. (2003); the MYSO sample is from Fayolle et al. (2015); and the hot-core sample is from Bisschop et al. (2007). Survival analysis was performed on the MYSO and hot-core samples in order to derive median values accounting for upper limits in these surveys.

We adopt beam-averaged abundances with respect to $\mathrm{CH}_{3} \mathrm{OH}$ under the assumption that the COMs and $\mathrm{CH}_{3} \mathrm{OH}$ have the same distributions (Herbst \& van Dishoeck 2009; Öberg et al. 2011b). This may not be an appropriate assumption, particularly for centrally concentrated warm emitters, but a lack of consistent structural information for the different sources prevents a more thorough treatment of beam dilution. There are some uncertainties then in the abundances with respect to $\mathrm{CH}_{3} \mathrm{OH}$ due to potential differences in the emission origin of each COM.

We now compare our LYSO sample with the hot corino IRAS 16293. We note that the LYSO sources in our survey could potentially host hot corinos, however our observations are not sensitive to this emission. The maximum observed transition energy of $\sim 40 \mathrm{~K}$ as well as the exclusion of line wings from spectral fitting support that the molecules observed are emitting from the outer regions of the protostar. Compared with IRAS 16293, the LYSOs are enhanced in the carbon chain $\mathrm{HC}_{3} \mathrm{~N}$, comparable or slightly enhanced in the cold $\mathrm{COMs} \mathrm{CH}_{2} \mathrm{CO}, \mathrm{CH}_{3} \mathrm{CHO}$, and $\mathrm{HNCO}$, and underabundant in the hot $\mathrm{COMS} \mathrm{CH}_{3} \mathrm{CN}, \mathrm{CH}_{3} \mathrm{OCH}_{3}$, and $\mathrm{CH}_{3} \mathrm{OCHO}$. This suggests an evolutionary sequence that results in the appearance of a central brightly emitting hot corino without destroying the envelope COM chemistry. We note that IRAS 16293 may not be representative of typical hot corino abundances, and a larger sample of these objects is required to make firm comparisons.

The MYSOs in Fayolle et al. (2015) have large envelopes with ice detections and lack a central hot core, and should therefore represent a comparable evolutionary stage to our LYSO sample. LYSOs have slightly higher abundances relative to MYSOs of the cold molecules $\mathrm{CH}_{3} \mathrm{CHO}$ and $\mathrm{HNCO}$, and comparable abundances for the warm molecules $\mathrm{CH}_{3} \mathrm{OCH}_{3}$ and $\mathrm{CH}_{3} \mathrm{CN}$. Overall, the chemistries of these types of objects appear very similar, with slight enhancements of cold molecules in the LYSOs that could be attributed to the generally colder temperatures of low-mass protostars.

It has been previously noted that hot corinos of LYSOs have abundances comparable to or greater than hot cores (e.g Bottinelli et al. 2007; Herbst \& van Dishoeck 
2009). While this is true for IRAS 16293, we note that from our unbiased sample of LYSOs this is clearly not typical for all low-mass objects. Furthermore, generalizing COMs as a single category seems to be an overly reductive classification, as cold and hot molecules represent distinct chemistries, and their emission trends between environments may differ.

\subsection{Comparisons with solar system chemistry}

Finally, we compare LYSO median abundances with observations of solar system comets (Crovisier et al. 2004; Mumma \& Charnley 2011; Goesmann et al. 2015). In Figure 13c, most cometary abundances appear consistent with LYSO abundances, with the exception of $\mathrm{HC}_{3} \mathrm{~N}$ and $\mathrm{CH}_{2} \mathrm{CO}$. Since LYSOs represent regions of solar-type star formation, this similarity supports the notion that (1) the solar system is fairly typical in its chemistry, and other sun-like stars should have similar chemical inventories; and (2) chemistry early in the evolution of a star is propagated through later stages. This suggests that some of the comet's molecular composition is inherited from the early stages of star formation.

$\mathrm{HC}_{3} \mathrm{~N}$ and $\mathrm{CH}_{2} \mathrm{CO}$ are each about an order of magnitude higher in LYSOs than in comets. The formation mechanisms discussed in Section 5.1 suggest that it may be difficult to form these molecules at later evolutionary stages: $\mathrm{HC}_{3} \mathrm{~N}$ is a dominantly gas-phase product favored by the low-density, cold outer envelope, while $\mathrm{CH}_{2} \mathrm{CO}$ is thought to form early via atom addition in simple ices. Both of these are also unsaturated molecules that are susceptible to depletion via hydrogenation or photoprocessing. This is in contrast to $\mathrm{CH}_{3} \mathrm{CHO}$ and $\mathrm{HNCO}$, which are also unsaturated but have formation channels involving radical recombinations that can continue at later stages of disk and planet formation.

\section{CONCLUSIONS}

Based on a survey of complex organic molecules towards 16 young low-mass protostars using the IRAM $30 \mathrm{~m}$ telescope, we conclude the following:

1. The molecules $\mathrm{CH}_{2} \mathrm{CO}, \mathrm{CH}_{3} \mathrm{CHO}, \mathrm{CH}_{3} \mathrm{OCH}_{3}$, $\mathrm{CH}_{3} \mathrm{OCHO}, \mathrm{HC}_{3} \mathrm{~N}$, and $\mathrm{HNCO}$ all have median column densities on the order of $10^{12} \mathrm{~cm}^{-2}$ and median abundances with respect to $\mathrm{CH}_{3} \mathrm{OH}$ around five to ten percent. $\mathrm{CH}_{3} \mathrm{CN}$ is an order of magnitude lower in column density and abundance.

2. For this sample of LYSOs at similar evolutionary stages, COM column densities span at least an order of magnitude. The distribution is reduced, especially for oxygen-bearing molecules, upon normalizaiton to $\mathrm{CH}_{3} \mathrm{OH}$.
3. Our findings are consistent with grain-surface chemistry as the dominant formation pathway to most COMs in this survey. There is evidence that the complex organics either form from or co-form with $\mathrm{CH}_{3} \mathrm{OH}$. While the nitrogen chemistry is regulated by additional factors, it appears still related to the $\mathrm{CH}_{3} \mathrm{OH}$ chemistry.

4. A warm-up model for low-mass protostars produces COM abundances in fair agreement with observations: the relative abundances of most molecules follow the same trend as seen observationally, though with a systematic underestimation due to an excess of $\mathrm{CH}_{3} \mathrm{OH}$ in the model. Most molecules are produced in the model by cold to lukewarm chemistry, consistent with observed rotational temperatures. $\mathrm{CH}_{3} \mathrm{CHO}$ is an exception, occurring at much higher temperatures in the model compared to observations. Gas-phase chemistry is responsible for lukewarm $\mathrm{CH}_{3} \mathrm{CN}$ production in the model, driven by high $\mathrm{HCN}$ abundances.

5. LYSO COM abundances are comparable to measurements in solar system comets, indicating that the sun is fairly typical among low-mass stars.

The study is based on observations with the IRAM $30 \mathrm{~m}$ Telescope. IRAM is supported by INSU/CNRS (France), MPG (Germany) and IGN (Spain). J.B.B acknowledges funding from the National Science Foundation Graduate Research Fellowship under Grant DGE1144152. K.I.O. acknowledges funding from the Simons Collaboration on the Origins of Life (SCOL) investigator award. 
7. APPENDIX: DETECTION SPECTRA 

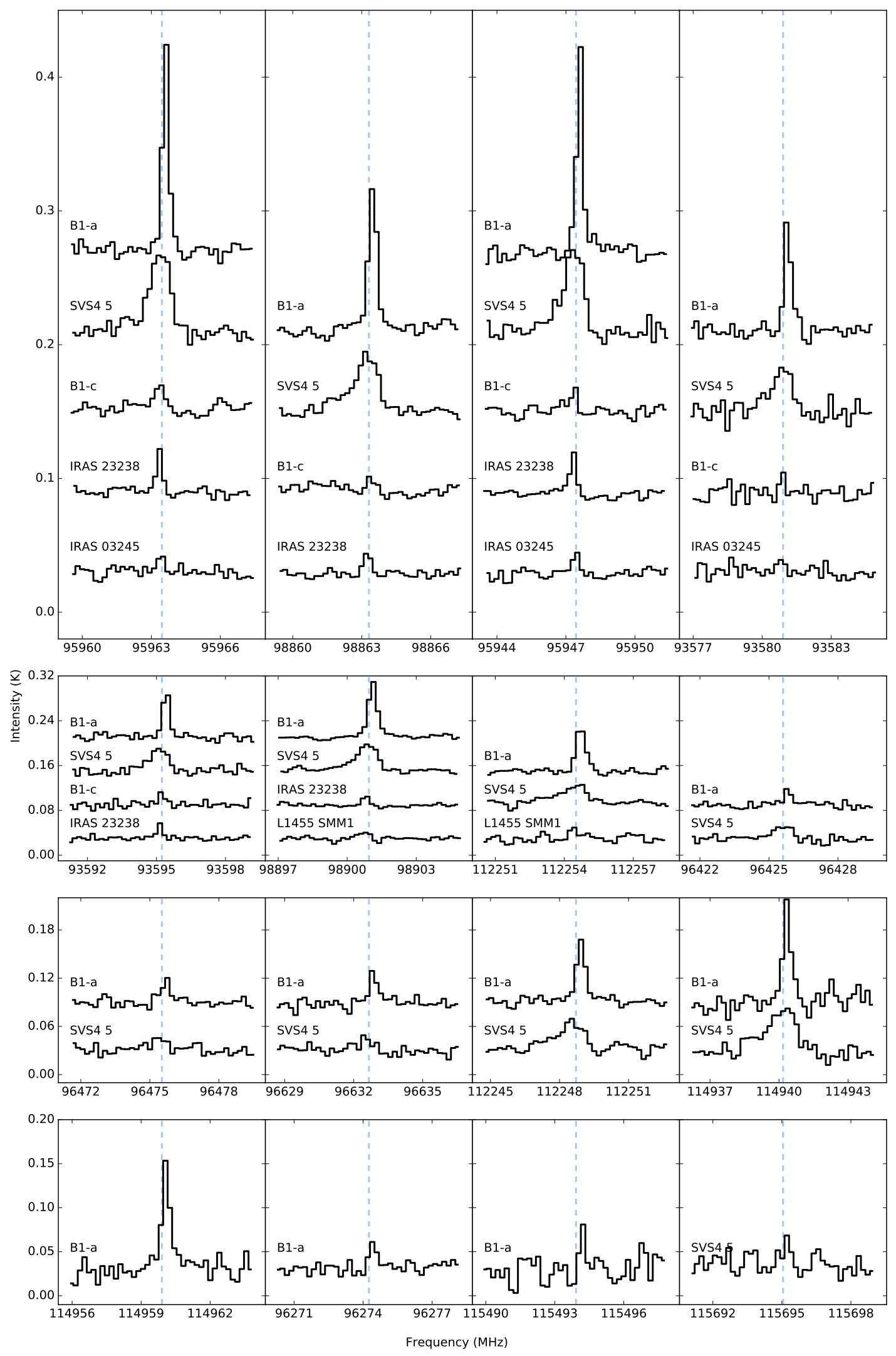

Figure 15. $\mathrm{CH}_{3} \mathrm{CHO}$ detected transitions. Grey dashed lines show line centers; spectra are offset for clarity. 


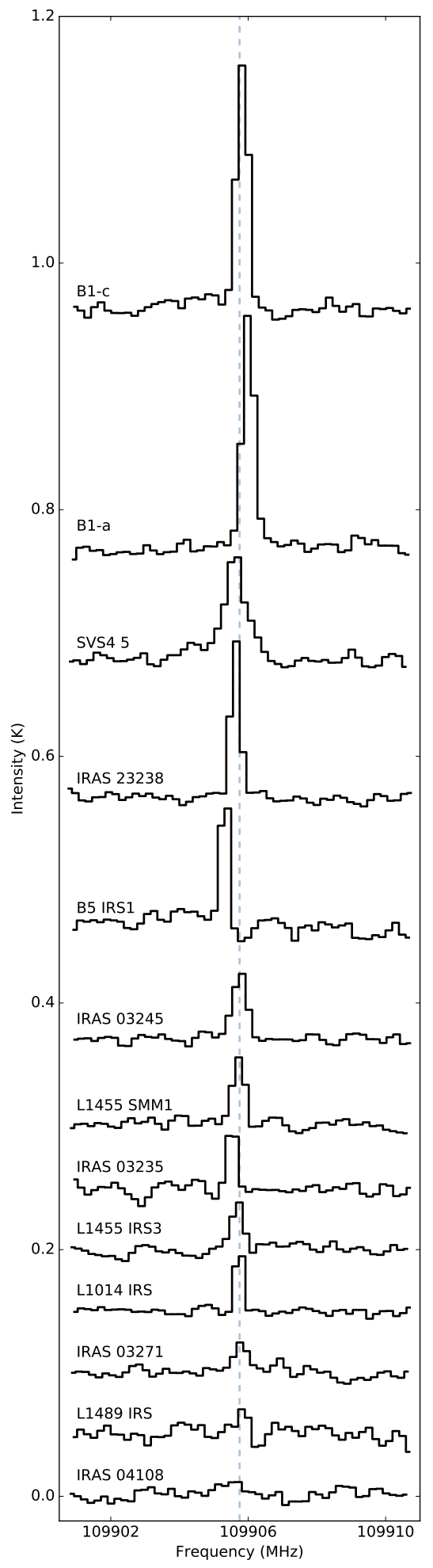

Figure 16. HNCO detected transitions. Grey dashed lines show line centers; spectra are offset for clarity.

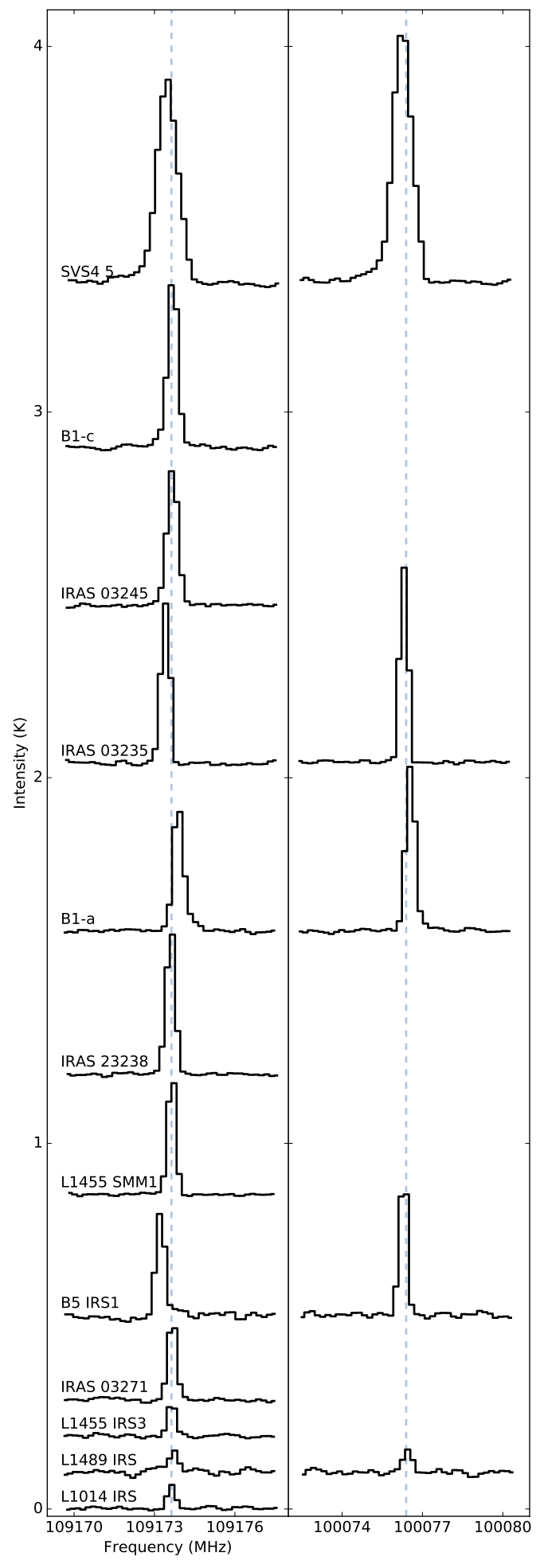

Figure 17. $\mathrm{HC}_{3} \mathrm{~N}$ detected transitions. Grey dashed lines show line centers; spectra are offset for clarity. 


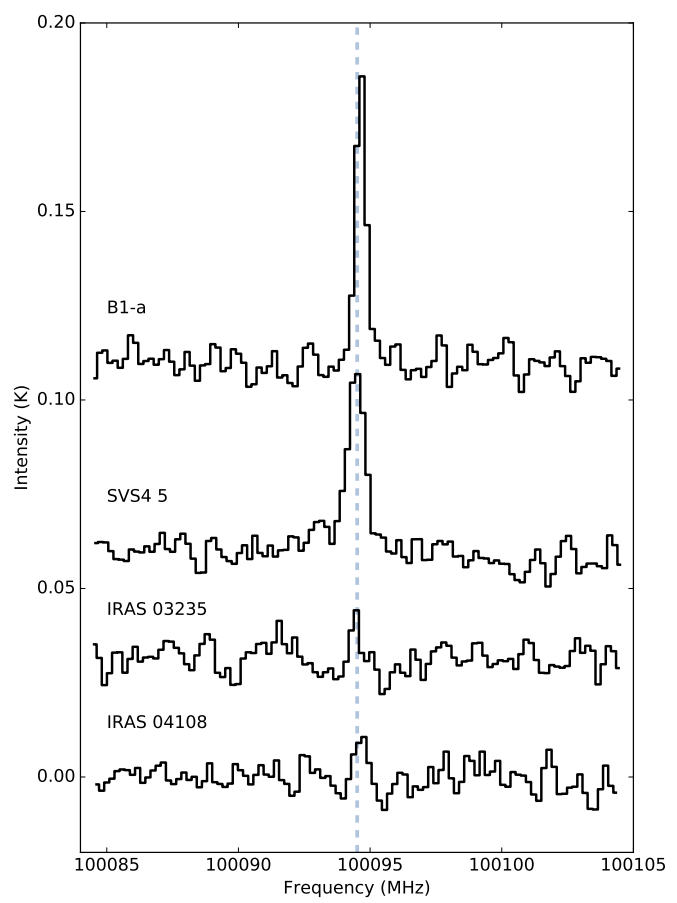

Figure 18. $\mathrm{CH}_{2} \mathrm{CO}$ detected transitions. Grey dashed lines show line centers; spectra are offset for clarity. 

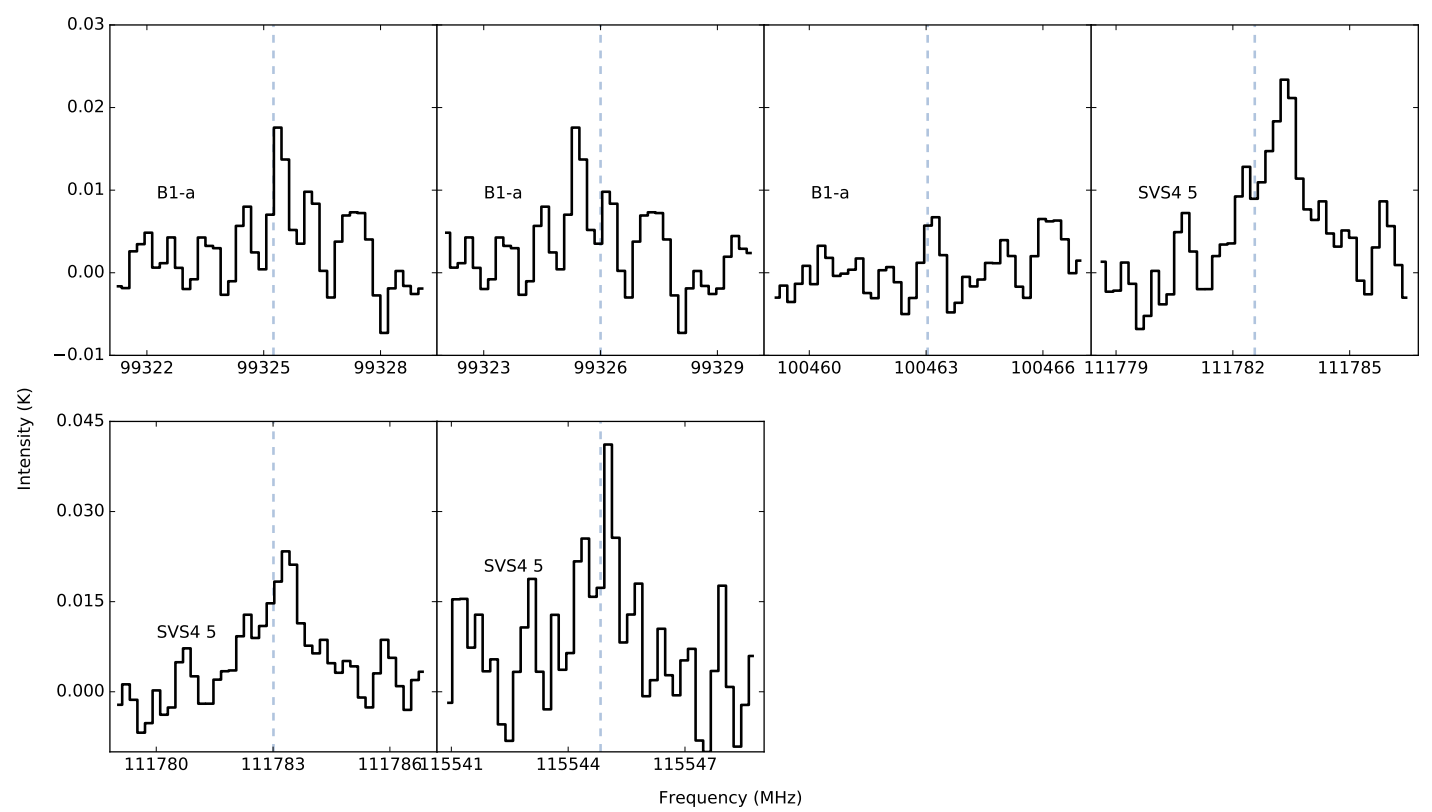

Figure 19. $\mathrm{CH}_{3} \mathrm{OCH}_{3}$ detected transitions. Grey dashed lines show line centers; spectra are offset for clarity.
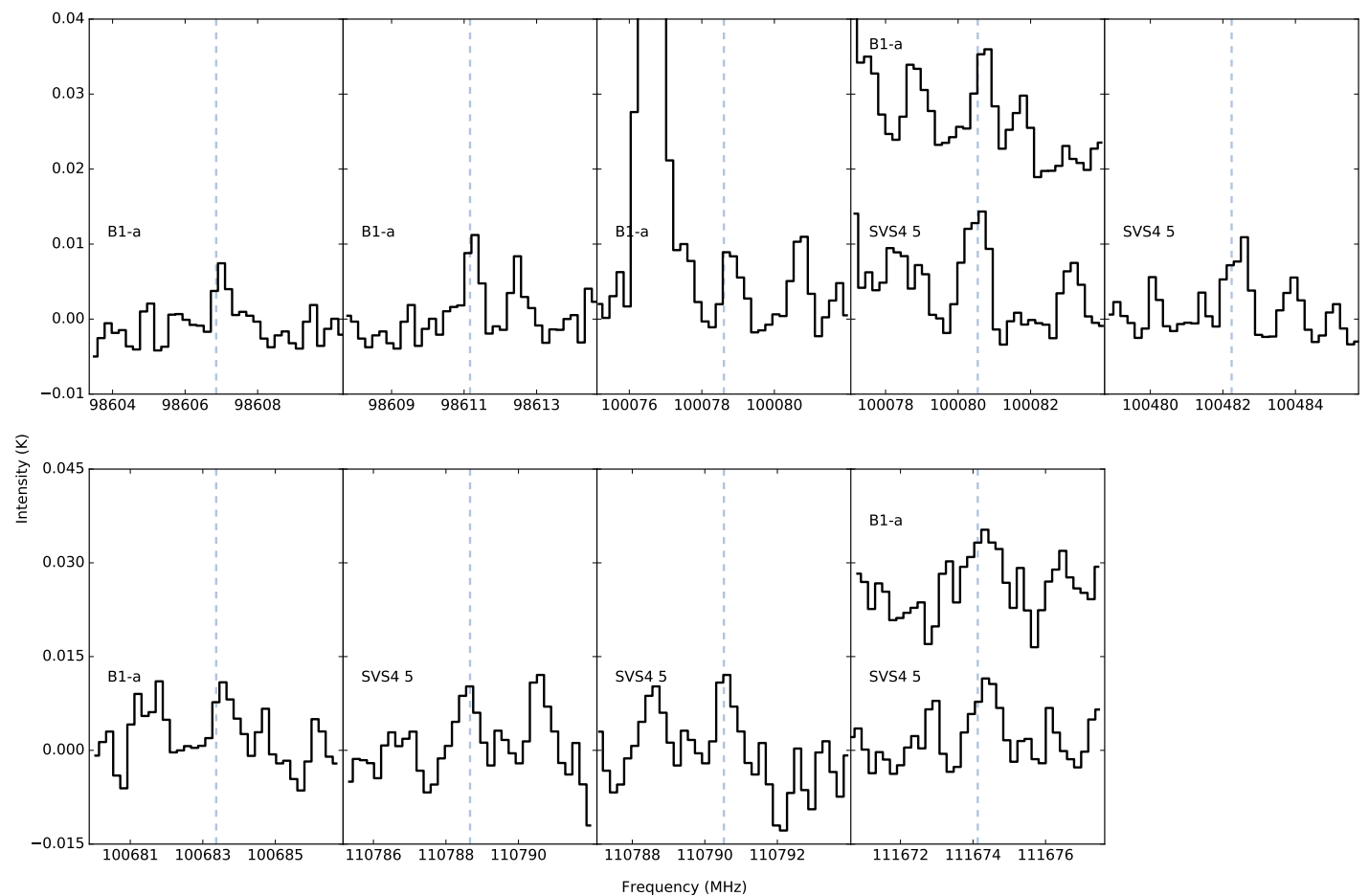

Figure 20. $\mathrm{CH}_{3} \mathrm{OCHO}$ detected transitions. Grey dashed lines show line centers; spectra are offset for clarity. 
8. APPENDIX: ROTATION DIAGRAMS 


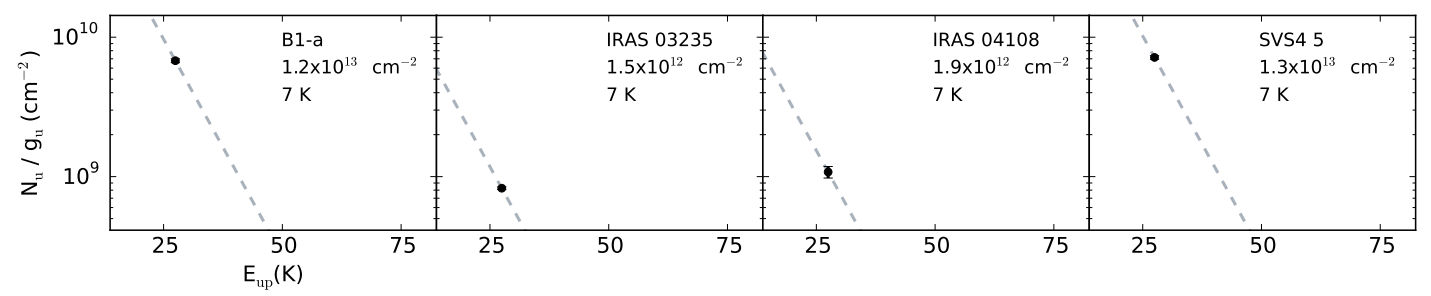

Figure 21. Rotation diagrams for $\mathrm{CH}_{2} \mathrm{CO}$. Black circles indicate detections and grey triangles indicate upper limits. Black dashed lines represent the fits to the data. When a line could not be fit, a rotational temperature was assumed as described in the text, shown in grey dashed lines.

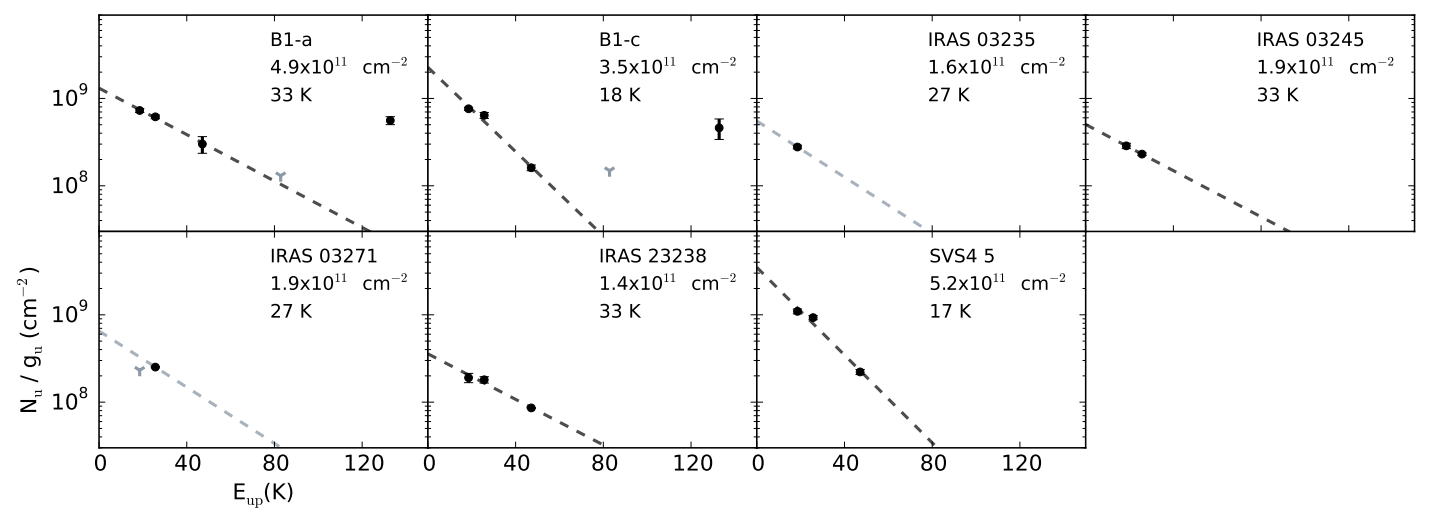

Figure 22. Rotation diagrams for $\mathrm{CH}_{3} \mathrm{CN}$. Black circles indicate detections and grey triangles indicate upper limits. Black dashed lines represent the fits to the data. When a line could not be fit, a rotational temperature was assumed as described in the text, shown in grey dashed lines.

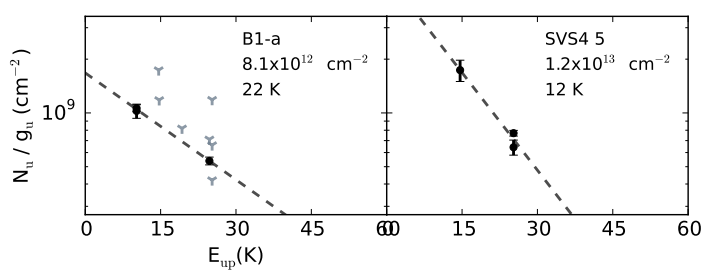

Figure 23. Rotation diagram for $\mathrm{CH}_{3} \mathrm{OCH}_{3}$. Black circles indicate detections and grey triangles indicate upper limits. Black dashed lines represent the fits to the data. When a line could not be fit, a rotational temperature was assumed as described in the text, shown in grey dashed lines.

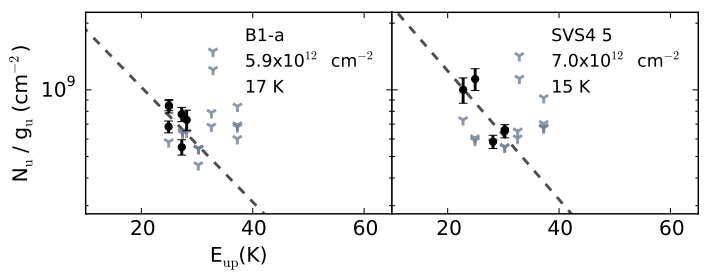

Figure 24. Rotation diagrams for $\mathrm{CH}_{3} \mathrm{OCHO}$. Black circles indicate detections and grey triangles indicate upper limits. Black dashed lines represent the fits to the data. When a line could not be fit, a rotational temperature was assumed as described in the text, shown in grey dashed lines. 


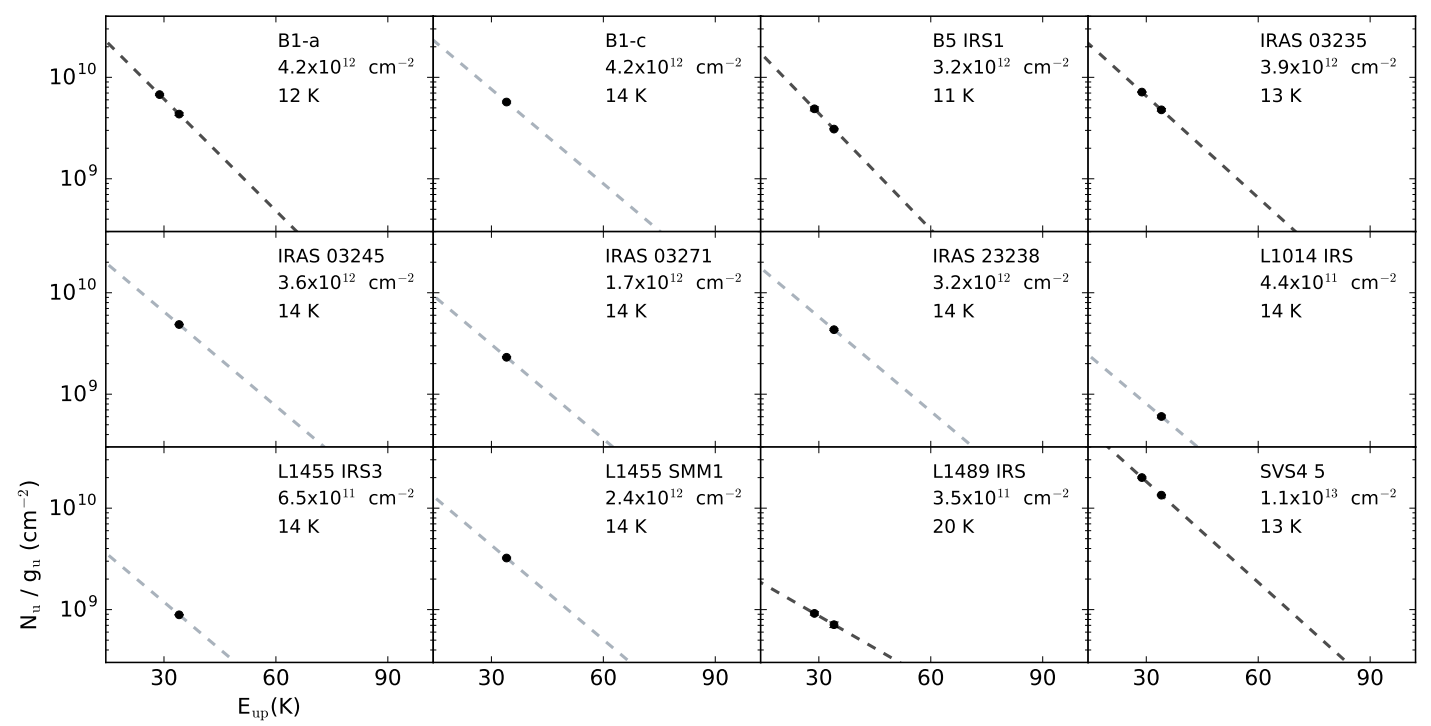

Figure 25. Rotation diagrams for $\mathrm{HC}_{3} \mathrm{~N}$. Black circles indicate detections and grey triangles indicate upper limits. Black dashed lines represent the fits to the data. When a line could not be fit, a rotational temperature was assumed as described in the text, shown in grey dashed lines.

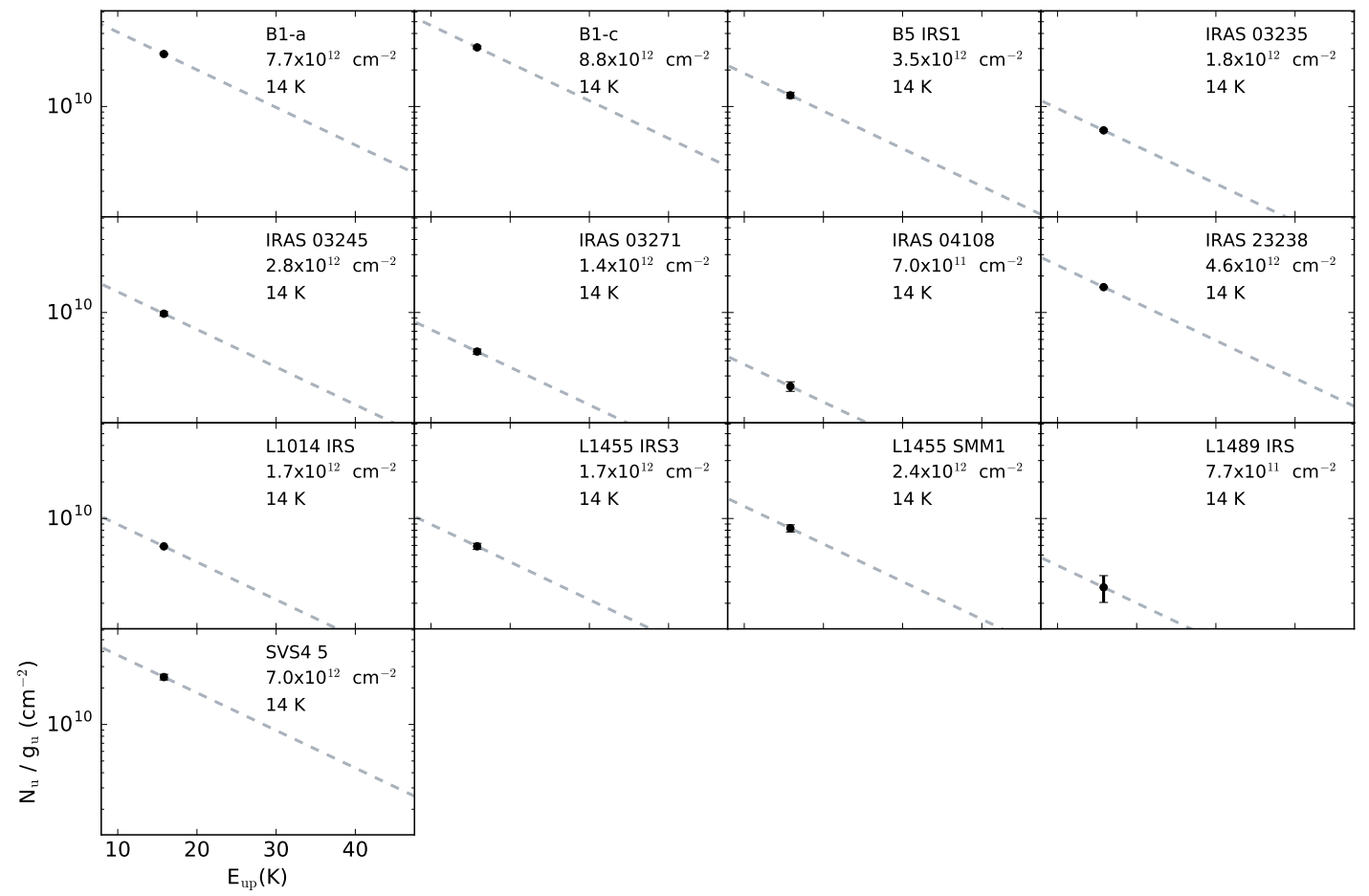

Figure 26. Rotation diagrams for HNCO. Black circles indicate detections and grey triangles indicate upper limits. Black dashed lines represent the fits to the data. When a line could not be fit, a rotational temperature was assumed as described in the text, shown in grey dashed lines. 


\section{APPENDIX: OBSERVED TRANSITIONS AND \\ UPPER LIMITS}

Table 6. Observed $\mathrm{CH}_{2} \mathrm{CO}$ line intensities

\begin{tabular}{|c|c|c|c|c|c|c|c|}
\hline \multirow[t]{2}{*}{$\begin{array}{l}\text { Freq. } \\
(\mathrm{GHz})\end{array}$} & \multirow[t]{2}{*}{ Transition } & \multicolumn{6}{|c|}{$\begin{array}{c}\int \mathrm{T}_{\mathrm{MB}} \mathrm{dV} \\
\left(\mathrm{K} \mathrm{km} \mathrm{s}^{-1}\right)\end{array}$} \\
\hline & & B1-a & SVS 4-5 & B5 IRS1 & IRAS 03235 & IRAS 04108 & L1489 IRS \\
\hline 100.095 & $5_{1,5}-4_{1,4}$ & $0.117(0.004)$ & $0.124(0.004)$ & $<0.006$ & $0.014(0.000)$ & $0.019(0.002)$ & $<0.006$ \\
\hline
\end{tabular}

Table 7. Observed $\mathrm{CH}_{3} \mathrm{CHO}$ line intensities

\begin{tabular}{|c|c|c|c|c|c|c|c|c|c|}
\hline \multirow[t]{2}{*}{$\begin{array}{l}\text { Freq. } \\
(\mathrm{GHz})\end{array}$} & \multirow[t]{2}{*}{ Transition } & \multicolumn{8}{|c|}{$\begin{array}{c}\int \mathrm{T}_{\mathrm{MB}} \mathrm{dV} \\
\left(\mathrm{K} \mathrm{km} \mathrm{s}^{-1}\right)\end{array}$} \\
\hline & & B1-a & SVS $4-5$ & B1-c & B5 IRS1 & HH 300 & IRAS 03235 & IRAS 03245 & IRAS 03254 \\
\hline 93.581 & $5_{1,5}-4_{1,4} \mathrm{~A}$ & $0.104(0.009)$ & $0.107(0.006)$ & - & - & - & - & - & - \\
\hline 93.595 & $5_{1,5}-4_{1,4} \mathrm{E}$ & $0.099(0.004)$ & $0.124(0.009)$ & $0.023(0.001)$ & - & - & - & - & - \\
\hline 95.947 & $5_{0,5}-4_{0,4} \mathrm{E}$ & $0.159(0.007)$ & $0.190(0.010)$ & - & - & - & - & $0.018(0.004)$ & - \\
\hline 95.963 & $5_{0,5}-4_{0,4} \mathrm{~A}$ & $0.172(0.005)$ & $0.197(0.011)$ & $0.033(0.001)$ & $<0.010$ & $<0.004$ & $<0.006$ & - & $<0.006$ \\
\hline 96.274 & $5_{2,4}-4_{2,3} \mathrm{~A}$ & $0.036(0.007)$ & $<0.011$ & - & - & - & - & - & - \\
\hline 96.426 & $5_{2,4}-4_{2,3} \mathrm{E}$ & $0.034(0.006)$ & $0.073(0.007)$ & - & - & - & - & - & - \\
\hline 96.476 & $5_{2,3}-4_{2,2} \mathrm{E}$ & $0.040(0.005)$ & $0.036(0.006)$ & - & - & - & - & - & - \\
\hline 96.633 & $5_{2,3}-4_{2,2} \mathrm{~A}$ & $0.053(0.011)$ & $0.035(0.000)$ & - & - & - & - & - & - \\
\hline 98.863 & $5_{1,4}-4_{1,3} \mathrm{E}$ & $0.148(0.004)$ & $0.146(0.007)$ & $0.013(0.003)$ & - & - & - & $<0.004$ & - \\
\hline 98.901 & $5_{1,4}-4_{1,3} \mathrm{~A}$ & $0.144(0.006)$ & $0.159(0.009)$ & $<0.006$ & - & - & - & $<0.003$ & - \\
\hline 112.249 & $6_{1,6}-5_{1,5} \mathrm{~A}$ & $0.098(0.005)$ & $0.108(0.009)$ & $<0.011$ & - & - & - & $<0.007$ & - \\
\hline 112.255 & $6_{1,6}-5_{1,5} \mathrm{E}$ & $0.110(0.005)$ & $0.104(0.008)$ & $<0.011$ & - & - & - & $<0.008$ & - \\
\hline 114.940 & $6_{0,6}-5_{0,5} \mathrm{E}$ & $0.135(0.011)$ & $0.204(0.011)$ & $<0.022$ & - & - & - & $<0.009$ & - \\
\hline 114.960 & $6_{0,6}-5_{0,5} \mathrm{~A}$ & $0.135(0.010)$ & $0.143(0.013)$ & $<0.018$ & - & - & - & - & - \\
\hline 115.494 & $6_{2,5}-5_{2,4} \mathrm{~A}$ & $0.038(0.009)$ & $<0.023$ & - & - & - & - & - & - \\
\hline 115.695 & $62,5-5_{2,4} \mathrm{E}$ & $0.071(0.005)$ & $<0.020$ & - & - & - & - & - & - \\
\hline 115.910 & $6_{2,4}-5_{2,3} \mathrm{E}$ & $<0.018$ & $<0.022$ & - & - & - & - & - & - \\
\hline \multirow[t]{2}{*}{116.118} & $6_{2,4}-5_{2,3} \mathrm{~A}$ & $<0.021$ & $0.059(0.004)$ & - & - & - & - & - & - \\
\hline & & IRAS 03271 & IRAS 04108 & IRAS 23238 & L1014 IRS & L1448 IRS1 & L1455 IRS3 & L1455 SMM1 & L1489 IRS \\
\hline 93.581 & $51,5-41,4 \mathrm{~A}$ & - & - & - & - & - & - & - & - \\
\hline 93.595 & $51,5-4_{1,4} \mathrm{E}$ & - & - & $0.025(0.000)$ & - & - & - & - & - \\
\hline 95.947 & $5_{0,5}-4_{0,4} \mathrm{E}$ & - & - & $0.035(0.004)$ & - & - & - & - & - \\
\hline 95.963 & $5_{0,5}-4_{0,4} \mathrm{~A}$ & $<0.004$ & $<0.007$ & $0.032(0.002)$ & $<0.003$ & $<0.004$ & $<0.004$ & - & $<0.008$ \\
\hline 98.863 & $51,4-4_{1,3} \mathrm{E}$ & - & - & $0.021(0.001)$ & - & - & - & - & - \\
\hline 98.901 & $51,4-4_{1,3} \mathrm{~A}$ & - & - & $0.020(0.000)$ & - & - & - & $0.025(0.004)$ & - \\
\hline 112.249 & $61,6-5_{1,5} \mathrm{~A}$ & - & - & $<0.006$ & - & - & - & $<0.008$ & - \\
\hline 112.255 & $61,6-5_{1,5} \mathrm{E}$ & - & - & $<0.005$ & - & - & - & $0.022(0.000)$ & - \\
\hline 114.940 & $60,6-5_{0,5} \mathrm{E}$ & - & - & $<0.008$ & - & - & - & $<0.013$ & - \\
\hline 114.960 & $6_{0,6}-5_{0,5} \mathrm{~A}$ & - & - & $<0.009$ & - & - & - & $<0.013$ & - \\
\hline 115.494 & $62,5-5_{2,4} \mathrm{~A}$ & - & - & - & - & - & - & $<0.015$ & - \\
\hline
\end{tabular}


Table 8. Observed $\mathrm{CH}_{3} \mathrm{CN}$ line intensities

\begin{tabular}{|c|c|c|c|c|c|c|c|c|c|}
\hline \multirow[t]{2}{*}{$\begin{array}{l}\text { Freq. } \\
(\mathrm{GHz})\end{array}$} & \multirow[t]{2}{*}{ Transition } & \multicolumn{8}{|c|}{$\begin{array}{c}\int \mathrm{T}_{\mathrm{MB}} \mathrm{dV} \\
\left(\mathrm{K} \mathrm{km} \mathrm{s}^{-1}\right)\end{array}$} \\
\hline & & B1-a & SVS 4-5 & B1-c & B5 IRS1 & HH 300 & IRAS 03235 & IRAS 03245 & IRAS 03254 \\
\hline 110.349 & $64-5_{4} 110.349$ & $6(4)-5(4)$ & $0.026(0.003)$ & - & $0.022(0.006)$ & - & - & - & - \\
\hline 110.364 & $6_{3}-5_{3} 110.364$ & $6(2)-5(2)$ & $<0.005$ & - & $<0.006$ & - & - & - & - \\
\hline 110.375 & $6_{2}-5_{2} 110.375$ & $6(1)-5(1)$ & $0.022(0.005)$ & $0.017(0.001)$ & $0.012(0.001)$ & - & - & - & - \\
\hline 110.381 & $6_{1}-5_{1} 110.381$ & $6(0)-5(0)$ & $0.050(0.002)$ & $0.076(0.004)$ & $0.052(0.004)$ & - & - & - & $0.019(0.001)$ \\
\hline \multirow[t]{2}{*}{110.383} & $6_{0}-5_{0} 110.383$ & $6(4)-5(4)$ & $0.061(0.003)$ & $0.092(0.006)$ & $0.064(0.003)$ & $<0.008$ & $<0.006$ & $0.023(0.001)$ & $0.024(0.002)$ \\
\hline & & IRAS 03271 & IRAS 04108 & IRAS 23238 & L1014 IRS & L1448 IRS1 & L1455 IRS3 & L1455 SMM1 & L1489 IRS \\
\hline 110.375 & $62-5_{2}$ & - & - & $0.006(0.000)$ & - & - & - & - & - \\
\hline 110.381 & $6_{1}-5_{1}$ & $0.021(0.000)$ & - & $0.015(0.001)$ & - & - & - & - & - \\
\hline 110.383 & $60-50$ & $<0.007$ & $<0.006$ & $0.016(0.002)$ & $<0.003$ & $<0.005$ & $<0.004$ & $<0.005$ & $<0.007$ \\
\hline
\end{tabular}

Table 9. Observed $\mathrm{CH}_{3} \mathrm{OCH}_{3}$ line intensities

\begin{tabular}{|c|c|c|c|c|c|c|c|c|c|}
\hline \multirow[t]{2}{*}{$\begin{array}{l}\text { Freq. } \\
(\mathrm{GHz})\end{array}$} & \multirow[t]{2}{*}{ Transition } & \multicolumn{8}{|c|}{$\begin{array}{c}\int \mathrm{T}_{\mathrm{MB}} \mathrm{dV} \\
\left(\mathrm{K} \mathrm{km} \mathrm{s}^{-1}\right)\end{array}$} \\
\hline & & B1-a & SVS $4-5$ & $\mathrm{~B} 1-\mathrm{c}$ & B5 IRS1 & HH 300 & IRAS 03235 & IRAS 03245 & IRAS 03254 \\
\hline 93.857 & $4_{2,3}-4_{1,4} \mathrm{EE}$ & $<0.005$ & - & - & - & - & - & - & - \\
\hline 96.850 & $5_{2,4}-5_{1,5} \mathrm{EE}$ & $<0.004$ & - & - & - & - & - & - & - \\
\hline 99.325 & $4_{1,4}-3_{0,3} \mathrm{EE}$ & $0.017(0.001)$ & - & - & - & - & - & - & - \\
\hline 99.326 & $4_{1,4}-3_{0,3} \mathrm{AA}$ & $0.011(0.001)$ & - & $<0.006$ & $<0.006$ & $<0.004$ & $<0.004$ & $<0.003$ & $<0.005$ \\
\hline 100.463 & $6_{2,5}-6_{1,6} \mathrm{EE}$ & $0.010(0.000)$ & - & - & - & - & - & - & - \\
\hline 100.466 & $6_{2,5}-6_{1,6}$ AA & $<0.003$ & - & - & - & - & - & - & - \\
\hline 111.783 & $7_{0,7}-6_{1,6} \mathrm{AA}$ & $<0.004$ & $0.012(0.001)$ & - & - & - & - & - & - \\
\hline 111.783 & $7_{0,7}-6_{1,6} \mathrm{EE}$ & $<0.004$ & $0.022(0.001)$ & - & - & - & - & - & - \\
\hline 111.784 & $7_{0,7}-7_{1,6} \mathrm{AE}$ & $<0.004$ & - & - & - & - & - & - & - \\
\hline \multirow[t]{2}{*}{115.545} & $5_{1,5}-4_{0,4} \mathrm{EE}$ & $<0.014$ & $0.041(0.006)$ & - & - & - & - & - & - \\
\hline & & IRAS 03271 & IRAS 04108 & IRAS 23238 & L1014 IRS & L1448 IRS1 & L1455 IRS3 & L1455 SMM1 & L1489 IRS \\
\hline 99.326 & $4_{1,4}-3_{0,3}$ AA & $<0.005$ & $<0.004$ & $<0.003$ & $<0.002$ & $<0.003$ & $<0.005$ & $<0.004$ & $<0.005$ \\
\hline
\end{tabular}

Table 10. Observed $\mathrm{CH}_{3} \mathrm{OCHO}$ line intensities

\begin{tabular}{|c|c|c|c|c|c|c|c|c|c|}
\hline \multirow[t]{2}{*}{$\begin{array}{l}\text { Freq. } \\
(\mathrm{GHz})\end{array}$} & \multirow[t]{2}{*}{ Transition } & \multicolumn{8}{|c|}{$\begin{array}{c}\int \mathrm{T}_{\mathrm{MB}} \mathrm{dV} \\
\left(\mathrm{K} \mathrm{km} \mathrm{s}^{-1}\right)\end{array}$} \\
\hline & & B1-a & SVS $4-5$ & B1-c & B5 IRS1 & HH 300 & IRAS 03235 & IRAS 03245 & IRAS 03254 \\
\hline 98.607 & $8_{3,6}-7_{3,5} \mathrm{E}$ & $0.012(0.001)$ & - & - & - & - & - & - & - \\
\hline 98.611 & $8_{3,6}-7_{3,5} \mathrm{~A}$ & $0.017(0.001)$ & - & $<0.008$ & $<0.007$ & $<0.005$ & $<0.003$ & $<0.005$ & $<0.004$ \\
\hline 100.079 & $9_{1,9}-8_{1,8} \mathrm{E}$ & $0.024(0.002)$ & $<0.007$ & - & - & - & - & - & - \\
\hline 100.081 & $9_{1,9}-8_{1,8} \mathrm{~A}$ & $0.024(0.001)$ & $0.032(0.004)$ & - & - & - & - & - & - \\
\hline 100.295 & $8_{3,5}-7_{3,4} \mathrm{E}$ & $<0.005$ & - & - & - & - & - & - & - \\
\hline 100.308 & $8_{3,5}-7_{3,4} \mathrm{~A}$ & $<0.005$ & - & - & - & - & - & - & - \\
\hline
\end{tabular}


Table 10 (continued)

\begin{tabular}{|c|c|c|c|c|c|c|c|c|c|}
\hline $\begin{array}{l}\text { Freq. } \\
(\mathrm{GHz})\end{array}$ & Transition & & & & $\begin{array}{r}\int \mathrm{T}_{\mathrm{N}} \\
(\mathrm{K} \mathrm{kr}\end{array}$ & $\begin{array}{l}\mathrm{B}_{\mathrm{B}} \mathrm{dV} \\
\left.\mathrm{s}^{-1}\right)\end{array}$ & & & \\
\hline 100.482 & $8_{1,7}-7_{1,6} \mathrm{E}$ & - & $0.025(0.003)$ & - & - & - & - & - & - \\
\hline 100.491 & $8_{1,7}-7_{1,6} \mathrm{~A}$ & - & $<0.006$ & - & - & - & - & - & - \\
\hline 100.682 & $9_{0,9}-8_{0,8} \mathrm{E}$ & $<0.006$ & $<0.006$ & - & - & - & - & - & - \\
\hline 100.683 & $9_{0,9}-8_{0,8} \mathrm{~A}$ & $0.019(0.001)$ & $<0.006$ & - & - & - & - & - & - \\
\hline 110.789 & $10_{1,10}-9_{1,9} \mathrm{E}$ & $<0.006$ & $0.023(0.000)$ & - & - & - & - & - & - \\
\hline 110.791 & $10_{1,10}-9_{1,9} \mathrm{~A}$ & $<0.006$ & $0.023(0.002)$ & - & - & - & - & - & - \\
\hline 110.880 & $9_{5,5}-8_{5,4} \mathrm{~A}$ & $<0.007$ & $<0.006$ & - & - & - & - & - & - \\
\hline 110.887 & $9_{3,7}-8_{3,6} \mathrm{~A}$ & $<0.006$ & $<0.006$ & - & - & - & - & - & - \\
\hline 111.170 & $10_{0,10}-9_{0,9} \mathrm{E}$ & $<0.006$ & $<0.006$ & - & - & - & - & - & - \\
\hline 111.172 & $10_{0,10}-9_{0,9} \mathrm{~A}$ & $<0.005$ & $<0.006$ & - & - & - & - & - & - \\
\hline 111.196 & $9_{4,6}-8_{4,5} \mathrm{~A}$ & $<0.005$ & $<0.006$ & - & - & - & - & - & - \\
\hline 111.223 & $9_{4,6}-8_{4,5} \mathrm{E}$ & $<0.006$ & $<0.007$ & - & - & - & - & - & - \\
\hline 111.408 & $9_{4,5}-8_{4,4} E$ & $<0.007$ & $<0.005$ & - & - & - & - & - & - \\
\hline 111.453 & $9_{4,5}-8_{4,4} \mathrm{~A}$ & $<0.006$ & $<0.006$ & - & - & - & - & - & - \\
\hline 111.674 & $9_{1,8}-8_{1,7} \mathrm{E}$ & $0.023(0.002)$ & $0.018(0.001)$ & - & - & - & - & - & - \\
\hline 111.682 & $9_{1,8}-8_{1,7} \mathrm{~A}$ & $<0.007$ & $<0.006$ & - & - & - & - & - & - \\
\hline 113.743 & $9_{3,6}-8_{3,5} \mathrm{E}$ & $<0.012$ & $<0.011$ & - & - & - & - & - & - \\
\hline 113.757 & $9_{3,6}-8_{3,5} \mathrm{~A}$ & $<0.014$ & $<0.014$ & - & - & - & - & - & - \\
\hline 116.545 & $9_{2,7}-8_{2,6} \mathrm{E}$ & $<0.029$ & $<0.025$ & - & - & - & - & - & - \\
\hline \multirow[t]{2}{*}{116.558} & $9_{2,7}-8_{2,6} \mathrm{~A}$ & $<0.033$ & $<0.027$ & - & - & - & - & - & - \\
\hline & & IRAS 03271 & IRAS 04108 & IRAS 23238 & L1014 IRS & L1448 IRS1 & L1455 IRS3 & L1455 SMM1 & L1489 IRS \\
\hline 98.611 & $83,6-73,5 \mathrm{~A}$ & $<0.004$ & $<0.005$ & $<0.003$ & $<0.003$ & $<0.003$ & $<0.004$ & $<0.004$ & $<0.005$ \\
\hline
\end{tabular}

Table 11. Observed $\mathrm{HC}_{3} \mathrm{~N}$ line intensities

\begin{tabular}{|c|c|c|c|c|c|c|c|c|c|}
\hline \multirow[t]{2}{*}{$\begin{array}{l}\text { Freq. } \\
(\mathrm{GHz})\end{array}$} & \multirow[t]{2}{*}{ Transition } & \multicolumn{8}{|c|}{$\begin{array}{c}\int \mathrm{T}_{\mathrm{MB}} \mathrm{dV} \\
\left(\mathrm{K} \mathrm{km} \mathrm{s}^{-1}\right)\end{array}$} \\
\hline & & B1-a & SVS $4-5$ & B1-c & B5 IRS1 & HН 300 & IRAS 03235 & IRAS 03245 & IRAS 03254 \\
\hline 100.076 & $11-10$ & $0.620(0.010)$ & $1.839(0.029)$ & - & $0.449(0.020)$ & - & $0.657(0.007)$ & - & - \\
\hline \multirow[t]{2}{*}{109.174} & $12-11$ & $0.474(0.014)$ & $1.467(0.019)$ & $0.624(0.014)$ & $0.337(0.006)$ & $<0.007$ & $0.522(0.015)$ & $0.531(0.004)$ & $<0.005$ \\
\hline & & IRAS 03271 & IRAS 04108 & IRAS 23238 & L1014 IRS & L1448 IRS1 & L1455 IRS3 & L1455 SMM1 & L1489 IRS \\
\hline 100.076 & $11-10$ & - & - & - & - & - & - & - & $0.084(0.003)$ \\
\hline 109.174 & $12-11$ & $0.253(0.004)$ & $<0.005$ & $0.472(0.009)$ & $0.066(0.002)$ & $<0.004$ & $0.097(0.001)$ & $0.352(0.007)$ & $0.078(0.005)$ \\
\hline
\end{tabular}

Table 12. Observed HNCO line intensities

\begin{tabular}{llllllllll}
\hline \hline $\begin{array}{c}\text { Freq. } \\
(\mathrm{GHz})\end{array}$ & Transition & & & & \multicolumn{2}{c}{$\begin{array}{c}\int \mathrm{T}_{\mathrm{MB}} \mathrm{dV} \\
\left(\mathrm{K} \mathrm{km} \mathrm{s}^{-1}\right)\end{array}$} & & & \\
\hline & & B1-a & SVS 4-5 & B1-c & B5 IRS1 & HH 300 & IRAS 03235 & IRAS 03245 & IRAS 03254 \\
\hline \hline 109.906 & $5_{0,5}-4_{0,4}$ & $0.228(0.002)$ & $0.191(0.010)$ & $0.247(0.007)$ & $0.097(0.005)$ & $<0.006$ & $0.050(0.001)$ & $0.076(0.003)$ & $<0.006$ \\
\hline & & IRAS 03271 & IRAS 04108 & IRAS 23238 & L1014 IRS & L1448 IRS1 & L1455 IRS3 & L1455 SMM1 & L1489 IRS \\
\hline
\end{tabular}


Table 12 (continued)

\begin{tabular}{|c|c|c|c|c|c|c|c|c|c|}
\hline $\begin{array}{l}\text { Freq. } \\
(\mathrm{GHz})\end{array}$ & Transition & & & & $\begin{array}{r}\int \mathrm{T}_{\mathrm{M}} \\
(\mathrm{K} \mathrm{km}\end{array}$ & $\begin{array}{l}{ }^{d V} \\
\left.s^{-1}\right)\end{array}$ & & & \\
\hline 109.906 & $5_{0,5}-4_{0,4}$ & $0.037(0.002)$ & $0.019(0.002)$ & $0.126(0.000)$ & $0.046(0.000)$ & $<0.004$ & $0.046(0.003)$ & $0.064(0.004)$ & $0.021(0.005)$ \\
\hline
\end{tabular}

\section{REFERENCES}

Arce, H. G., Santiago-García, J., Jørgensen, J. K., Tafalla, M., \& Bachiller, R. 2008, ApJL, 681, L21

Bacmann, A., Taquet, V., Faure, A., Kahane, C., \& Ceccarelli, C. 2012, A\&A, 541, L12

Balucani, N., Ceccarelli, C., \& Taquet, V. 2015, MNRAS, 449, L16

Belloche, A., Meshcheryakov, A. A., Garrod, R. T., et al. 2017, arXiv:1701.04640, A\&Ain press

Bennett, C. J., Jamieson, C. S., Osamura, Y., \& Kaiser, R. I. 2005, ApJ, 624, 1097

Bisschop, S. E., Jørgensen, J. K., Bourke, T. L., Bottinelli, S., \& van Dishoeck, E. F. 2008, A\&A, 488, 959

Bisschop, S. E., Jørgensen, J. K., van Dishoeck, E. F., \& de Wachter, E. B. M. 2007, A\&A, 465, 913

Blake, G. A., Sutton, E. C., Masson, C. R., \& Phillips, T. G. 1987, ApJ, 315, 621

Boogert, A. C. A., Pontoppidan, K. M., Knez, C., et al. 2008, ApJ, 678, 985

Bottinelli, S., Ceccarelli, C., Williams, J. P., \& Lefloch, B. 2007, A\&A, 463, 601

Bottinelli, S., Ceccarelli, C., Lefloch, B., et al. 2004a, ApJ, 615, 354

Bottinelli, S., Ceccarelli, C., Neri, R., et al. 2004b, ApJL, 617, L69

Bottinelli, S., Boogert, A. C. A., Bouwman, J., et al. 2010, ApJ, 718, 1100

Brinch, C., Crapsi, A., Jørgensen, J. K., Hogerheijde, M. R., \& Hill, T. 2007, A\&A, 475, 915

Caselli, P., \& Ceccarelli, C. 2012, A\&A Rv, 20, 56

Cazaux, S., Tielens, A. G. G. M., Ceccarelli, C., et al. 2003, ApJL, 593, L51

Cernicharo, J., Marcelino, N., Roueff, E., et al. 2012, ApJL, 759, L43

Chandler, C. J., \& Richer, J. S. 2000, ApJ, 530, 851

Crovisier, J., Bockelée-Morvan, D., Colom, P., et al. 2004, A\&A, 418,1141

Enoch, M. L., Corder, S., Dunham, M. M., \& Duchêne, G. 2009, ApJ, 707, 103

Fayolle, E. C., Öberg, K. I., Garrod, R. T., van Dishoeck, E. F., \& Bisschop, S. E. 2015, A\&A, 576, A45

Fedoseev, G., Chuang, K.-J., van Dishoeck, E. F., Ioppolo, S., \& Linnartz, H. 2016, MNRAS, 460, 4297

Fedoseev, G., Ioppolo, S., Zhao, D., Lamberts, T., \& Linnartz, H. 2015, MNRAS, 446, 439

Feigelson, E. D., \& Nelson, P. I. 1985, ApJ, 293, 192

Furlan, E., McClure, M., Calvet, N., et al. 2008, ApJS, 176, 184

Garrod, R. T. 2013, ApJ, 765, 60
Garrod, R. T., Belloche, A., Mueller, H. S. P., \& Menten, K. M. 2017, arXiv:1701.07160, A\&Ain press

Garrod, R. T., \& Herbst, E. 2006, A\&A, 457, 927

Garrod, R. T., Wakelam, V., \& Herbst, E. 2007, A\&A, 467, 1103

Garrod, R. T., Widicus Weaver, S. L., \& Herbst, E. 2008, ApJ, 682,283

Goesmann, F., Rosenbauer, H., Bredehöft, J. H., et al. 2015, Science, 349, doi:10.1126/science.aab0689

Goldsmith, P. F., \& Langer, W. D. 1999, ApJ, 517, 209

Graninger, D. M., Wilkins, O. H., \& Öberg, K. I. 2016, ApJ, 819,140

Hatchell, J., Fuller, G. A., Richer, J. S., Harries, T. J., \& Ladd, E. F. 2007, A\&A, 468, 1009

Herbst, E., \& van Dishoeck, E. F. 2009, ARA\&A, 47, 427

Hudson, R. L., \& Loeffler, M. J. 2013, ApJ, 773, 109

Jørgensen, J. K., Schöier, F. L., \& van Dishoeck, E. F. 2002, A\&A, 389, 908

Maity, S., Kaiser, R. I., \& Jones, B. M. 2014, ApJ, 789, 36

Miller, R., Gong, G., \& Muñoz, A. 1981, Survival Analysis, A Wiley-Interscience publication (Wiley)

Mumma, M. J., \& Charnley, S. B. 2011, ARA\&A, 49, 471

Öberg, K. I. 2016, ChRv, 116(17), 9631

Öberg, K. I., Boamah, M. D., Fayolle, E. C., et al. 2013, ApJ, 771,95

Öberg, K. I., Boogert, A. C. A., Pontoppidan, K. M., et al. 2011a, ApJ, 740, 109

Öberg, K. I., Bottinelli, S., Jørgensen, J. K., \& van Dishoeck, E. F. 2010, ApJ, 716, 825

Öberg, K. I., Garrod, R. T., van Dishoeck, E. F., \& Linnartz, H. 2009, A\&A, 504, 891

Öberg, K. I., Lauck, T., \& Graninger, D. 2014, ApJ, 788, 68

Öberg, K. I., van der Marel, N., Kristensen, L. E., \& van Dishoeck, E. F. 2011b, ApJ, 740, 14

Pontoppidan, K. M., van Dishoeck, E. F., \& Dartois, E. 2004, A\&A, 426, 925

Schöier, F. L., van der Tak, F. F. S., van Dishoeck, E. F., \& Black, J. H. 2005, A\&A, 432, 369

van der Tak, F. F. S., Black, J. H., Schöier, F. L., Jansen, D. J. \& van Dishoeck, E. F. 2007, A\&A, 468, 627

van Dishoeck, E. F., Blake, G. A., Jansen, D. J., \& Groesbeck, T. D. 1995, ApJ, 447, 760

Visser, R., Doty, S. D., \& van Dishoeck, E. F. 2011, A\&A, 534, A132

Visser, R., van Dishoeck, E. F., Doty, S. D., \& Dullemond, C. P. 2009, A\&A, 495, 881 